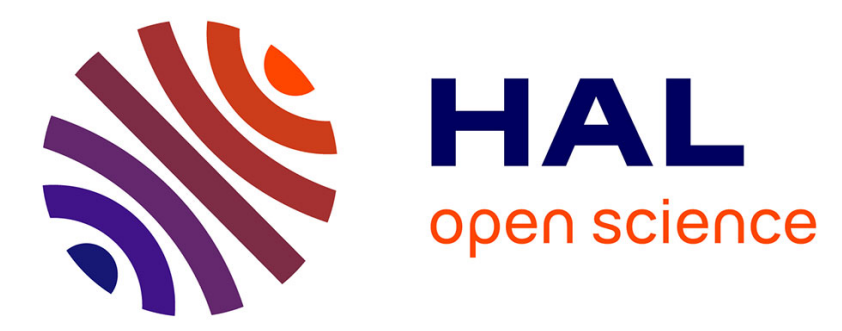

\title{
Propagation of acoustic waves in two waveguides coupled by perforations. II. Analysis of periodic lattices of finite length
}

Marc Pachebat, Jean Kergomard

\section{- To cite this version:}

Marc Pachebat, Jean Kergomard. Propagation of acoustic waves in two waveguides coupled by perforations. II. Analysis of periodic lattices of finite length. Acta Acustica united with Acustica, 2016, 102, pp.611-625. 10.3813/AAA.918978 . hal-01286174v2

\section{HAL Id: hal-01286174 \\ https://hal.science/hal-01286174v2}

Submitted on 7 Jun 2016

HAL is a multi-disciplinary open access archive for the deposit and dissemination of scientific research documents, whether they are published or not. The documents may come from teaching and research institutions in France or abroad, or from public or private research centers.
L'archive ouverte pluridisciplinaire HAL, est destinée au dépôt et à la diffusion de documents scientifiques de niveau recherche, publiés ou non, émanant des établissements d'enseignement et de recherche français ou étrangers, des laboratoires publics ou privés. 


\title{
Propagation of acoustic waves in two waveguides coupled by perforations. II. Analysis of periodic lattices of finite length
}

\author{
Marc Pachebat, Jean Kergomard, \\ LMA, CNRS, UPR 7051, Aix-Marseille Univ, Centrale Marseille, \\ 13453 Marseille Cedex 13, France. pachebat@lma.cnrs-mrs.fr
}

\section{Summary}

The paper deals with the generic problem of two waveguides coupled by perforations, which can be perforated tube mufflers without or with partitions, possibly with absorbing materials. Other examples are ducts with branched resonators of honeycomb cavities, which can be coupled or not, and splitter silencers. Assuming low frequencies, only one mode is considered in each guide. The propagation in the two waveguides can be very different, thanks e.g. to the presence of constrictions. The model is a discrete, periodic one, based upon 4th-order impedance matrices and their diagonalization. All the calculation is analytical, thanks to the partition of the matrices in 2nd-order matrices, and allows the treatment of a very wide types of problems. Several aspects are investigated: the local or non-local character of the reaction of one guide to the other; the definition of a coupling coefficient; the effect of finite size when a lattice with $n$ cells in inserted into an infinite guide; the ${ }^{45}$ relationship between the transmission coefficient and the dispersion. The assumptions are as follows: linear acoustics, no mean flow, rigid wall. However the effect of the series impedance of the perforations, which is generally ignored, is taken into account, and is dis- ${ }^{5}$ cussed. When there are no losses, it is shown that, for symmetry reasons, the cut-off frequencies depend on either the series impedance or the shunt admittance, and are the eigenfrequencies of the cells of the lattice, with zero-pressure or zero-velocity at the ends of the ${ }^{55}$ cells.

\section{Introduction}

The present paper describes an attempt of a generic study of several problems that are now classical: per-

35 forated tube mufflers without [1-5] or with partitions [6]. They can be with absorbing materials [7-9]. Related problems are tubes with branched 65 resonators, which can be uncoupled $[10,11]$ or coupled [12], or with honeycomb cavities $[13,14]$. Other

40 kind of systems are splitter silencers with perforated facing $[15-20]$. This generic problem is that of a periodic lattice of two waveguides coupled by perforations. 70

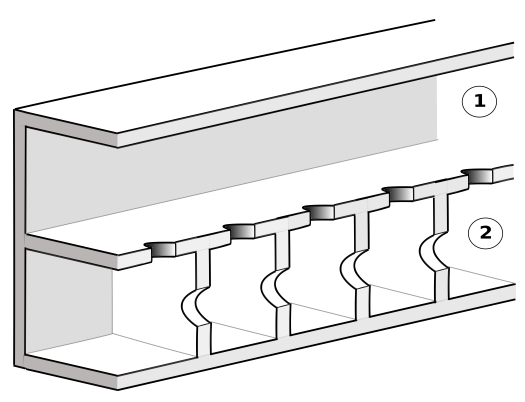

Figure 1: Sectional view of the type of lattice under study : lateral perforations (dark grey) couple two waveguides periodically along the direction of propagation. Each waveguide have its own acoustic properties. In Guide 2, diaphragms are periodically spaced along the direction of propagation

Assuming low frequencies, only one mode is considered in each guide, therefore the system in study is a system with two coupled modes. The propagation in the two waveguides can be very different, thanks to the presence of constrictions, diaphragms, porous material, partitions or other type of obstacles (see Figure 1). Following Sullivan [2], we use a discrete, periodic model based on 4th-order transfer matrices. However in a transfer matrix, after diagonalization, there are terms like $\cosh (n \Gamma)$, where $n$ is the number of cells and $\Gamma$ is the real propagation constant when the modes are evanescent. This leads to diverging terms when $n$ tends to infinity, and can be avoided by combining a decoupling approach, i.e., a diagonalization, and then the transformation of a transfer matrix into an impedance matrix for the finite-length lattice, which involves terms like $\tanh (n \Gamma)$, which cannot diverge. ${ }_{60}$ A decoupling approach was used also in a continuous modeling ( $[4,21]$, see also [22] p 356).

The papers aims at showing that it is possible to use an analytical formulation for a very wide class of problems, with the illustration of basic examples of coupled waveguides. Thanks to a discrete model, the diagonalization of 4th-order transfer matrices can be found analytically, by using the partition of these matrices into 2nd-order matrices. The study of the coupling between two guides especially involves an analysis of the local or non-local character of the coupling. 
Generally speaking, coupling is obviously strong when the perforations are wide, but also when propagation in the two guides is rather similar (i.e., the two propagation constants are close). This analysis is done for attices of finite length, focusing on the behaviour relationships between the transmission coefficient and the dispersion curves and frequency bands.

A major difficulty is the modeling of the perforations. Semi-empirical formulas are generally used 2,23-27], especially when there is a mean flow. One focus of the present paper is on the role of the series impedance of the perforation [28], which can be ignored in a continuous model, but not in a discrete model. A priori this impedance, due to the antisymmetric field in the perforation, must be accounted for the case of wide and well spaced perforations. To our knowledge, no paper used the complete model found in the paper published in 1994 [28]. However for a similar problem in musical acoustics, the effect of the series impedance of tone-holes of woodwind instruments, can be significant [29-31].

The values of the perforation shunt admittance and series impedance are not discussed in detail in this paper, but the values given in [28] are sufficient for a (exact values were given for the-2D, rectangular case at low frequencies, but for the cylindrical case, only approximate formulas were proposed).

Several papers are concerned with more general systems with more than two guides, or with $2 \mathrm{D}$ silencers, in particular for applications to metamaterials [10,32-38]. They are not discussed here. Concern- ${ }_{130}$ ing a general view on $1 \mathrm{D}$ periodic structures, we refer to classical references $[39,40]$.

The assumptions are as follows: linear acoustics, no mean flow, rigid walls. However the diagonalization is done in a very wide, linear framework. The basic ${ }^{135}$ geometry and the model used are described in Section 2, with the definition of the transfer matrix of a lattice cell. Section 3 derives the eigenvalues and eigenvectors of a cell, using the more general result given in Appendix A. For the case of lossless guides, ${ }^{140}$ the cut-off frequencies are determined.

For a finite lattice of $n$ cells, the impedance matrix is derived by using the calculation of the transfer matrix calculated in Appendix B. Finally the transmission coefficient of the lattice into an infinite waveguide is derived. Section 5 proposes a theoretical analysis of the coupling between the two guides, focusing on the effect of the series impedance; on a definition of a coupling coefficient; and on a derivation of a condition ${ }_{150}$ for a local reaction. Finally numerical simulations of application examples are presented in Section 6, with an analysis of the transmission coefficient with respect to the nature of the two modes. It is shown how the extreme values of the coupling coefficient.

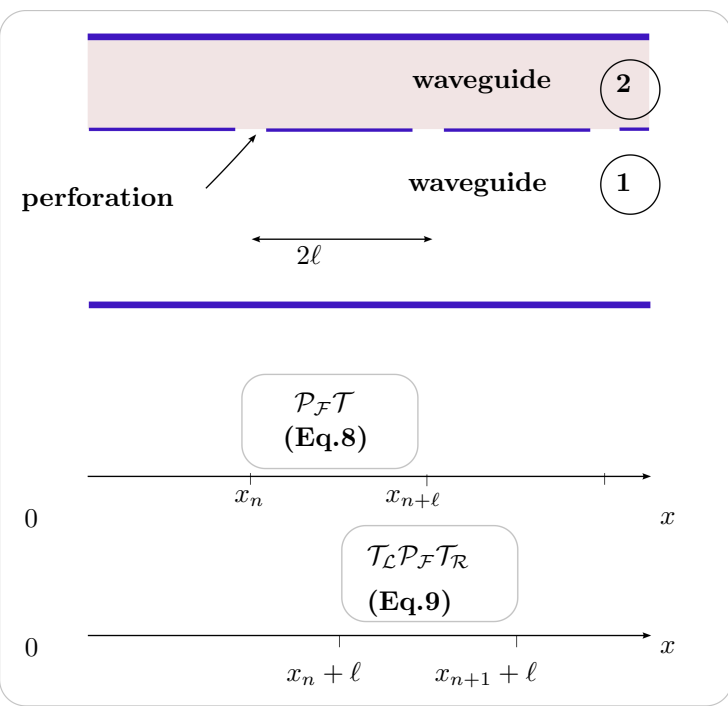

Figure 2: Basic geometry; an asymmetric cell includes one perforation followed by a length $2 \ell$ of tube, with the transfer matrix $P \mathcal{T}$ between abscissas $x_{n}$ and $x_{n+1}$, while a symmetric cell includes one perforation between two lengths $\ell$ of tube, with the transfer ma$\operatorname{trix} T_{L} \mathcal{P}_{\mathcal{F}} \mathcal{T}_{\mathcal{R}}$, between abscissas $x_{n}+\ell$ and $x_{n+1}+\ell$.

\section{Generic geometry; model and notations}

\section{$2.1 \quad$ Geometry}

The two guides are coupled by perforation, as shown in Fig. 2. When their cross section is uniform, the waves are planar at an axial distance from perforations larger that the transverse dimensions, so that the evanescent modes due to perforations vanish. When the cross section is not uniform, the change in cross-section area needs to be sufficiently far from the perforation, i.e., at an axial distance larger than the transverse dimension. The propagation in the guides is characterized by the effective density $\rho_{i}$ and the speed of sound $c_{i}$ (the subscript $i=1,2$ ). The change in cross section allows various situations to be created, as shown in Figure 3. When the propagation is identical in the two guides, the lattice is homogeneous, while when diaphragms are present in one guide only, the lattice is non-homogeneous. The case of branched resonators without longitudinal coupling between them is a limit case, with a local reaction of Guide 2 on Guide 1. In the following the term "(non)-uniform" will be used for each waveguide and the term "(non-)homogeneous" will be used for the lattice.

\subsection{Model for a perforation}

The general model, valid in harmonic regime, is developed in [28]. It is summarized hereafter, with similar 
notations. Four basic quantities are chosen to be the coefficients $p$ and $v$ of the planar mode for the acoustic pressure and velocity, respectively, in the two guides. They build a 4 th-order vector, $\mathcal{V}$, as follows:

$$
\mathcal{V}=\left(\begin{array}{l}
\mathbf{V}_{1} \\
\mathbf{V}_{2}
\end{array}\right) \text { where } \mathbf{V}_{i}=\left(\begin{array}{c}
p_{i} \\
v_{i}
\end{array}\right)
$$

$i=1,2$. The following notations are chosen: callicharacters correspond to 4th-order matrices and vectors, while bold characters correspond to 2ndorder matrices and vectors (e.g. $\mathcal{I}$ and $\mathbf{I}$ are the identity matrices of order 4 and 2, respectively); other quantities are scalar. For a periodic medium made ofymetric lattice cells, one perforation at $x_{n}$ is followed by a portion of length $2 \ell$ of separated waveguides (see Fig. 2). The vectors $\mathcal{V}$ are related by 4 thorder matrices.

For a perforation, the following relationship is dein [28] (the subscripts L and $\mathrm{R}$ correspond to the left side and right side of a perforation, respectively):

$$
\begin{aligned}
\mathcal{V}_{L}=\mathcal{P}_{\mathcal{F}} \mathcal{V}_{R} \\
\text { where } \quad \mathcal{P}_{\mathcal{F}}=\left(\begin{array}{cc}
\left(\gamma_{1}+\gamma_{2} \mathbf{M}\right) & \gamma_{2}(\mathbf{I}-\mathbf{M}) \\
\gamma_{1}(\mathbf{I}-\mathbf{M}) & \left(\gamma_{2}+\gamma_{1} \mathbf{M}\right)
\end{array}\right) \\
\text { with } \gamma_{1,2}=\frac{S_{1,2}}{S_{1}+S_{2}}, \\
\mathbf{M}=\mathbf{I}+\frac{2 Z_{a} Y_{s}}{1-Z_{a} Y_{s}} \mathbf{K}, \quad \mathbf{K}=\left(\begin{array}{cc}
1 & Y_{s}^{-1} \\
Z_{a}^{-1} & 1
\end{array}\right) .
\end{aligned}
$$

$S_{1,2}$ are the cross-section areas of the guides. This model considers the effect of a perforation as localized at the abscissa of the perforation center, as explained in [41]. $Z_{a}$ and $Y_{s}$ are the series impedance and shunt admittance of the perforation, respectively ${ }^{195}$ (these quantities are specific impedance and specific admittance, i.e., ratios pressure/velocity and velocity/pressure, respectively). Both are acoustic masses and correspond to the anti-symmetric and symmetric pressure field in the perforation, respectively. When $Z_{a}=0, Y_{s}$ produces a jump in velocity inside each guide, from the left to the right of the perforation, along the guide axis. In a dual way, when $Y_{s}=0, Z_{a}$ produces a jump in pressure inside each guide, from the left to the right of the perforation. Reciprocity is assumed, therefore the determinant of $\mathcal{P}_{\mathcal{F}}$ is unity.

\subsection{Model for the propagation in the waveguides}

For a non-perforated portion of the waveguides, between abscissas $x_{n}$ and $x_{n+1}$, the following 4th-order ${ }^{210}$ matrix relationship is written as:

$$
\mathcal{V}_{R, n}=\mathcal{T} \mathcal{V}_{L, n+1} \text { where } \mathcal{T}=\left(\begin{array}{cc}
\mathbf{T}_{1} & \mathbf{0} \\
\mathbf{0} & \mathbf{T}_{2}
\end{array}\right)
$$

The general transfer matrices

$$
\mathbf{T}_{1,2}=\left(\begin{array}{ll}
A_{1,2} & B_{1,2} \\
C_{1,2} & D_{1,2}
\end{array}\right)
$$

a.

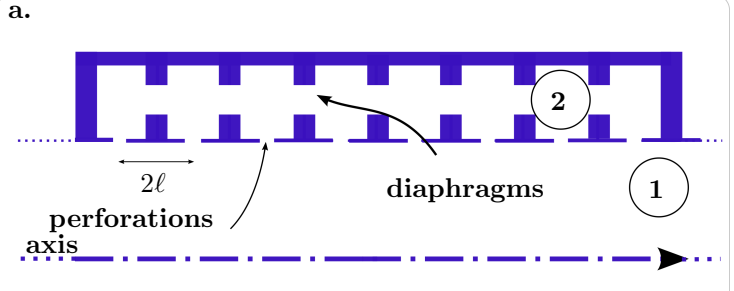

Non-homogeneous lattice

b.

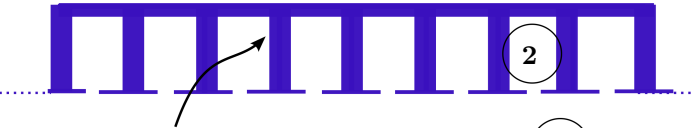

rigid walls $\left(\sigma_{d}=0\right)$

1

axis

Branched Helmholtz resonators

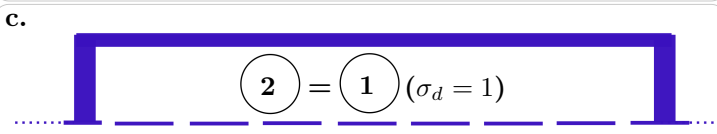

1

axis

Homogeneous lattice

Figure 3: (a) Three simple cases of coupled waveguides. Non-homogeneous lattice, (b) branched Helmholtz resonators, and (c) homogeneous lattice. Case (b) is a limit case of perfectly local reaction.

$(i=1,2)$ are of 2 nd-order and describe the propagation within Guides 1 and 2 . The coefficients $B_{1,2}$ are specific impedances, while the coefficients $C_{1,2}$ are specific admittances. In the separated portion, the geometry may be various, e.g., may includes discontinuities and/or dissipation. Provided that the perforation shunt admittance and the series impedance are known, and with the restricting assumption of an equivalent fluid model, the presence of porous material within the lattice can be described by the model.

\subsection{Model for the propagation with perforations: asymmetric and symmetric cells}

For a periodic medium, two types of cells can be considered (see Fig. 2): i) an asymmetric cell, involving a perforation followed by a portion of tubes of length $2 \ell$; ii) a symmetric cell involving a portion of tubes of length $\ell$, then a perforation, then a portion of tube of length $\ell$. The complete transfer matrix of an asymmetric cell (see Fig.2) can be characterized by the equation:

$$
\mathcal{V}_{L, n}=\mathcal{P}_{\mathcal{F}} \mathcal{T} \mathcal{V}_{L, n+1}
$$

The case of a symmetric cell is more particular, but remains very general. It will be used for the diago- 
nalization (see next section). For such a cell, between ${ }_{250}$ abscissas $x_{n}+\ell$ and $x_{n+1}+\ell$, the transfer matrix relationship is given by:

$$
\mathcal{V}_{n-1}=\left(\mathcal{T}_{\mathcal{L}} \mathcal{P}_{\mathcal{F}} \mathcal{T}_{\mathcal{R}}\right) \mathcal{V}_{n}
$$

where $\mathcal{V}_{n}=^{t}\left(p_{1 n}, v_{1 n}, p_{2 n}, v_{2 n}\right)$, defined in Equation ${ }^{255}$ $(1)$, is considered at mid-distance (abscissa $x_{n}+\ell$ ) between two perforations. The transfer matrix $\mathcal{T}$ (Equation (6)) describing the uncoupled propagation over distance $2 \ell$ between two neighboring perforations is therefore the product of the two transfer matrices:

$$
\mathcal{T}=\mathcal{T}_{\mathcal{R}} \mathcal{T}_{\mathcal{L}}
$$

where $\mathcal{T}_{\mathcal{L}}$ (resp. $\left.\mathcal{T}_{\mathcal{R}}\right)$ describes the uncoupled propagation over the distance $\ell$ situated on the left (resp. right) of one perforation. Since $\mathcal{T}$ is block-diagonal (Equation (6)), we can adopt the same decomposi$\mathbf{T}_{2}=\mathbf{T}_{R 2} \mathbf{T}_{L 2}$. Moreover, in order for the cell to be symmetric, we generalize the concept of reversed fourterminal explained in [39]. The matrices $\mathbf{T}_{L}(i=1,2)$ need to be proportional to the invert of the matrices $\mathbf{T}_{R}$, with a change in sign for the x-axis, and with the same determinant $\delta$. This means:

$$
\left(\begin{array}{cc}
A_{R} & B_{R} \\
C_{R} & D_{R}
\end{array}\right)=\left(\begin{array}{cc}
D_{L} & B_{L} \\
C_{L} & A_{L}
\end{array}\right),
$$

With this condition the matrix $\mathbf{T}$ is symmetric:

$$
A=D=A_{L} D_{L}+B_{L} C_{L} ; B=2 B_{L} D_{L} C=2 C_{L} A_{L} .
$$

Moreover reciprocity is assumed (in particular no flow is present), i.e., the determinant is unity, as well as the determinant of the 4 th-order matrix $\mathcal{T}$. More general cases are investigated in Appendix A.

\section{Infinite periodic lattice: ${ }^{265}$ eigenvalues and eigenvectors}

\subsection{Eigenvalues and eigenvectors}

In this section, we are searching for the diagonal form ${ }^{270}$ of the transfer matrix $\mathcal{T}_{L} \mathcal{P}_{\mathcal{F}} \mathcal{T}_{\mathcal{R}}$ (Equation (9)) for an elementary, symmetric cell (with reciprocity) of the periodic lattice shown on Fig.(2):

$$
\mathcal{T}_{\mathcal{L}} \mathcal{P}_{\mathcal{F}} \mathcal{T}_{\mathcal{R}} \stackrel{\text { def }}{=} \mathcal{E} \mathcal{D} \mathcal{E}^{-1}
$$

with

$$
\begin{aligned}
& \mathcal{D}=\left(\begin{array}{cccc}
\lambda^{(1)} & 0 & 0 & 0 \\
0 & \lambda^{(2)} & 0 & 0 \\
0 & 0 & \lambda^{(3)} & 0 \\
0 & 0 & 0 & \lambda^{(4)}
\end{array}\right) \text {, and } \\
& \mathcal{E}=\left(\mathcal{W}^{(1)} \mathcal{W}^{(2)} \mathcal{W}^{(3)} \mathcal{W}^{(4)}\right) \text {. }
\end{aligned}
$$

$\lambda^{(i)}$ are the eigenvalues and $\mathcal{W}^{(i)}(i=1 . .4)$ are the eigenvectors. The detailed calculation is derived in Appendix Afor the most general case (no reciprocity is required). Since for the perforation matrix (Equation $(2))$, $\operatorname{det} \mathcal{P}_{\mathcal{F}}=1$, the eigenvalues of the diagonal matrix $\mathcal{D}$ (Equation (14)) can be grouped by inverse pairs when reciprocity holds for the elementary cell $\left(\lambda^{(1)}, \lambda^{(2)}\right)=(\lambda, 1 / \lambda)$ and $\left(\lambda^{(3)}, \lambda^{(4)}\right)=\left(\lambda^{\prime}, 1 / \lambda^{\prime}\right)$ (see [28]). Each pair corresponds to opposite propagation directions of an eigenmode along the lattice axis. They are denoted $\lambda=\exp (\Gamma)$ and $\lambda^{\prime}=\exp \left(\Gamma^{\prime}\right)$. This leads to the following dispersion equation for the unknowns $\Gamma$ and $\Gamma^{\prime}$ :

$$
\operatorname{det} \mathbf{T}_{0}=A_{0} D_{0}-B_{0} C_{0}=0,
$$

where

$$
\begin{aligned}
& A_{0}=D_{0}=-\sinh \Gamma\left[\gamma_{2} / Q_{1}+\gamma_{1} / Q_{2}\right] \\
& B_{0}=\frac{1}{Y_{s}}-\gamma_{2} B_{1} / Q_{1}-\gamma_{1} B_{2} / Q_{2} \\
& C_{0}=\frac{1}{Z_{a}}-\gamma_{2} C_{1} / Q_{1}-\gamma_{1} C_{2} / Q_{2}
\end{aligned}
$$

with $Q_{i}=\left(\cosh \Gamma-A_{i}\right)$. The eigenvector matrix is found to be:

$$
\mathcal{E}=v_{0}\left(\begin{array}{cccc}
z_{1} & z_{1} & z_{1}^{\prime} & z_{1}^{\prime} \\
h_{1} & -h_{1} & h_{1}^{\prime} & -h_{1}^{\prime} \\
-z_{2} & -z_{2} & -z_{2}^{\prime} & -z_{2}^{\prime} \\
-h_{2} & +h_{2} & -h_{2}^{\prime} & +h_{2}^{\prime}
\end{array}\right) \widetilde{\mathcal{D}}^{-1 / 2}
$$

$$
\begin{aligned}
z_{1} & =\frac{1}{\gamma_{1} Q_{1}}\left[B_{R 1} \cosh (\Gamma / 2)-w_{0} D_{R 1}(\sinh \Gamma / 2)\right](1 \\
h_{1} & =\frac{1}{\gamma_{1} Q_{1}}\left[A_{R 1}(\sinh \Gamma / 2)-w_{0} C_{R 1} \cosh (\Gamma / 2)\right](20) \\
w_{0} & =B_{0} / A_{0}=D_{0} / C_{0} .
\end{aligned}
$$

$v_{0}$ is an arbitrary constant with the dimension of a velocity. Similar expressions can be found for $h_{2}$ and $z_{2}$. For $h_{1}^{\prime}$ and $z_{1}^{\prime}, \Gamma$ is changed in $\Gamma^{\prime}$ and $w_{0}$ in $w_{0}^{\prime}$, and similarly for the quantities with subscript 2 . It is shown in Appendix $\mathrm{C}$ that because of the reciprocity property the following relationships are induced :

$$
\gamma_{1} z_{1} h_{1}^{\prime}=-\gamma_{2} z_{2} h_{2}^{\prime} ; \gamma_{1} z_{1}^{\prime} h_{1}=-\gamma_{2} z_{2}^{\prime} h_{2} .
$$

The matrix $\widetilde{\mathcal{D}}^{-1 / 2}$ corresponds to a shift of an eigenvector by one half-cell. Notice that because the eigenvectors are defined apart from a multiplicative constant, three quantities define an eigenvector. Coming back to the definition of the physical-quantity vectors (see Equation (1)), we deduce the following interpretations:

- The ratio $z_{1} / h_{1}$ is the (specific) characteristic impedance in Guide 1 for the first propagation constant $\Gamma$; 
- Because the second eigenvalue corresponds to a change in sign of the propagation constant $\Gamma$, the corresponding characteristic impedance is $-z_{1} / h_{1}$, as expected;

In order to calculate the constant $\Gamma$, Equation (16) can be re-written as a 2nd-order equation for the unknown $\cosh (\Gamma)$. For this purpose the terms proportional to $\gamma_{1}^{2}$ and $\gamma_{2}^{2}$ can be rearranged by using the re- ${ }^{320}$ lations $\gamma_{1}+\gamma_{2}=1$ and $\sinh ^{2} \Gamma-B_{i} C_{i}=\cosh ^{2} \Gamma-A_{i}^{2}$. The following equation is obtained:

$$
\begin{gathered}
\left(1-Y_{s} Z_{a}\right) \cosh ^{2} \Gamma+ \\
-\left[A_{1}+A_{2}+\gamma_{2} E_{1}+\gamma_{1} E_{2}+d_{12}\right] \cosh \Gamma \\
+\gamma_{1} \gamma_{2} Y_{s} Z_{a}\left[\left(B_{1} C_{2}+B_{2} C_{1}\right)+2+2 A_{1} A_{2}\right] \\
+A_{1} A_{2}\left(1+Y_{s} Z_{a}\right)+\gamma_{2} A_{2} E_{1}+\gamma_{1} A_{1} E_{2}=0
\end{gathered}
$$

where $d_{12}=\left(A_{1}-A_{2}\right)\left(\gamma_{2}-\gamma_{1}\right) Y_{s} Z_{a}$ and $E_{i}={ }_{330}$ $Y_{s} B_{i}+Z_{a} C_{i}$. Thanks to Equation (23), general solutions $\cosh \Gamma$ and $\cosh \Gamma^{\prime}$, for the two modes $\Gamma$ and $\Gamma^{\prime}$ of the lattice can be written explicitly.

\subsection{Lossless lattices; cut-off frequen- ${ }^{33}$ cies}

Up to now the considered lattice is can be lossy, when one of the coefficients defining a cell is complex. For lossless waveguides, several types of waves can exist [28]. When reciprocity holds, each of the two modes with propagation constant $\Gamma$ and $\Gamma^{\prime}$ can be either propagating or evanescent. In the case of two evanescent waves the possibility for the propagation constant to be complex was found: the energy flux in each guide decreases exponentially, but is not zero (its sign is opposite in the two guides, ensuring the energy conservation).

Ref. [28] studied the particular case of an homogeneous lattice, i.e., a lattice with identical transfer matrices $\mathbf{T}$ in the two guides. This happens for example when the guides are straight guides with the same sound speed and density. In this case, there is at least one propagating wave, and the decomposition of the propagation into two modes (one is planar, the other one is called the "flute" mode) is valid even for a lattice with irregular perforations.

The cut-off frequencies are given by $\cosh \Gamma= \pm 1$, i.e., $\Gamma=0$ or $\Gamma=j \pi$. Therefore, according to Equations (17), $A_{0}=D_{0}=0$, and the dispersion equation (16) implies either $B_{0}=0$ or $C_{0}=0$. Writing $\cosh \Gamma= \pm 1$ in Equation (17), and using Equations
$(11,12)$ with the property $\operatorname{det}\left(\mathbf{T}_{L}\right)=1$, the cut-off frequencies are given by one of the four following equations:

$$
\begin{gathered}
\Gamma=0 ; B_{0}=0 ; \Rightarrow \frac{1}{Y_{s}}+\gamma_{2} \frac{D_{L 1}}{C_{L 1}}+\gamma_{1} \frac{D_{L 2}}{C_{L 2}}=0 ; \\
\Gamma=0 ; C_{0}=0 ; \Rightarrow \frac{1}{Z_{a}}+\gamma_{2} \frac{A_{L 1}}{B_{L 1}}+\gamma_{1} \frac{A_{L 2}}{B_{L 2}}=0 ; \\
\Gamma=j \pi ; B_{0}=0 ; \Rightarrow \frac{1}{Y_{s}}+\gamma_{2} \frac{B_{L 1}}{A_{L 1}}+\gamma_{1} \frac{B_{L 2}}{A_{L 2}}=0 ; \\
\Gamma=\mathrm{j} \pi ; C_{0}=0 ; \Rightarrow \frac{1}{Z_{a}}+\gamma_{2} \frac{C_{L 1}}{D_{L 1}}+\gamma_{1} \frac{C_{L 2}}{D_{L 2}}=0 .
\end{gathered}
$$

The characteristic impedances of the two guides, $z_{i} / h_{i}$ (see Equations. $(19,20)$, are found to be either infinite or zero. It is interesting to interpret these results. Consider the example of Equation (24). Because $\Gamma=0$, for an infinite lattice, $p_{n}=p_{n+1}$ in each guide, and because the characteristic impedance is infinite, the velocity vanishes at the extremities of the cell. Consequently, if there is a opening at the ex325 tremity of the cell, this cut-off does not depend on the opening. It can be checked that this equation gives the eigenfrequency of the cell when it is closed at their extremities (infinite impedance). The second and the third terms of Equation (24) correspond to the impedance in Guide 1 and 2, respectively, at the abscissa of the perforation, calculated by projecting the infinite impedance at the end of the cell to the perforation abscissa. Moreover the pressure field in the cell being symmetrical, the series impedance $Z_{a}$ does not intervene.

Similar interpretation can be done for the three other equations, using the duality pressure/velocity.

\section{Impedance matrix of a lattice on $n$ cells; insertion into an infinite waveguide}

\subsection{Impedance matrix}

In order to derive the (acoustic) impedance matrix of a lattice of $n$ cells, the vector $\mathcal{V}$ (Equation (1)) is replaced by a vector $\widetilde{\mathcal{V}}$ defined as follows:

$$
\widetilde{\mathcal{V}}=\left(\begin{array}{c}
\mathbf{P} \\
\mathbf{U}
\end{array}\right) \quad \text { where } \mathbf{P}=\left(\begin{array}{l}
p_{1} \\
p_{2}
\end{array}\right) \text { and } \mathbf{U}=\left(\begin{array}{l}
u_{1} \\
u_{2}
\end{array}\right) \text {, }
$$

where $u_{i}=S_{i} v_{i}(i=1,2)$ are the flow rates. In Appendix A3 it is shown that for these vectors the transfer matrix relationship can be written as:

$$
\widetilde{\mathcal{V}}_{0}=\left(\begin{array}{ll}
\mathbf{Z} & \mathbf{0} \\
\mathbf{0} & \mathbf{G}
\end{array}\right)\left(\begin{array}{ll}
\mathbf{C}_{\mathbf{n}} & \mathbf{S}_{\mathbf{n}} \\
\mathbf{S}_{\mathbf{n}} & \mathbf{C}_{\mathbf{n}}
\end{array}\right)\left(\begin{array}{ll}
\mathbf{Z} & \mathbf{0} \\
\mathbf{0} & \mathbf{G}
\end{array}\right)^{-1} \widetilde{\mathcal{V}}_{n},
$$

with

$$
\mathbf{C}_{n}=\left(\begin{array}{cc}
\cosh n \Gamma & 0 \\
0 & \cosh n \Gamma^{\prime}
\end{array}\right)
$$




$$
\begin{gathered}
\mathbf{S}_{n}=\left(\begin{array}{cc}
\sinh n \Gamma & 0 \\
0 & \sinh n \Gamma^{\prime}
\end{array}\right), \\
\mathbf{Z}=\left(\begin{array}{cc}
z_{1} & z_{1}^{\prime} \\
-z_{2} & -z_{2}^{\prime}
\end{array}\right), \mathbf{G}=\left(\begin{array}{cc}
g_{1} & g_{1}^{\prime} \\
-g_{2} & -g_{2}^{\prime}
\end{array}\right),
\end{gathered}
$$

if $g_{i}=S_{i} h_{i}$. This acoustic impedance matrix is directly derived from this transfer matrix. It is chosen for two reasons: i) as explained hereafter, the impedance matrix avoids numerical difficulties that appear using transfer matrix products, for strongly evanescent eigenmodes and a large number of cells; ii) the impedance matrix makes easy the boundary conditions to be introduced at each end of the lattice. Consider two 4 th-order vectors ${ }^{t}\left(\mathbf{P}_{\mathbf{0}} \mathbf{U}_{0}\right)$ and ${ }_{380}$ ${ }^{t}\left(\mathbf{P}_{\mathbf{n}} \mathbf{U}_{n}\right)$ related by a (general) matrix as follows:

$$
\left(\begin{array}{l}
\mathbf{P}_{\mathbf{0}} \\
\mathbf{U}_{\mathbf{0}}
\end{array}\right)=\left(\begin{array}{ll}
\mathbf{A} & \mathbf{B} \\
\mathbf{C} & \mathbf{D}
\end{array}\right)\left(\begin{array}{l}
\mathbf{P}_{\mathbf{n}} \\
\mathbf{U}_{n}
\end{array}\right)
$$

where $\mathbf{A}, \mathbf{B}, \mathbf{C}$ and $\mathbf{D}$ are 2 nd order-matrices. This ${ }^{385}$ expression is equivalent to:

$$
\left(\begin{array}{l}
\mathbf{P}_{\mathbf{0}} \\
\mathbf{P}_{n}
\end{array}\right)=\left(\begin{array}{cc}
\mathbf{A} \mathbf{C}^{-1} & {\left[\mathbf{B}-\mathbf{A} \mathbf{C}^{-1} \mathbf{D}\right]} \\
\mathbf{C}^{-\mathbf{1}} & -\mathbf{C}^{-1} \mathbf{D}
\end{array}\right)\left(\begin{array}{l}
\mathbf{U}_{\mathbf{0}} \\
\mathbf{U}_{n}
\end{array}\right)
$$

Applying this result to the transfer matrix (Equation (29)), the impedance matrix of the lattice of $n$ cells is obtained:

$$
\begin{gathered}
\left(\begin{array}{l}
p_{1,0} \\
p_{2,0} \\
p_{1, n} \\
p_{2, n}
\end{array}\right)=\mathcal{Z}\left(\begin{array}{cc}
\mathbf{C}_{n} \mathbf{S}_{n}^{-1} & -\mathbf{S}_{n}^{-1} \\
\mathbf{S}_{n}^{-1} & -\mathbf{S}_{n}^{-1} \mathbf{C}_{n}
\end{array}\right) \mathcal{G}^{-1}\left(\begin{array}{l}
u_{1,0} \\
u_{2,0} \\
u_{1, n} \\
u_{2, n}
\end{array}\right) \\
\text { where } \mathcal{Z}=\left(\begin{array}{cc}
\mathbf{Z} & \mathbf{0} \\
\mathbf{0} & \mathbf{Z}
\end{array}\right) \text { and } \mathcal{G}=\left(\begin{array}{cc}
\mathbf{G} & \mathbf{0} \\
\mathbf{0} & \mathbf{G}
\end{array}\right),
\end{gathered}
$$

where the identity $\left[\mathbf{S}_{\mathrm{n}}-\mathbf{C}_{\mathrm{n}} \mathbf{S}_{\mathrm{n}}^{-1} \mathbf{C}_{\mathrm{n}}\right]=-\mathbf{S}_{\mathrm{n}}^{-1}$ is used. Actually, because of the different sign before $\mathbf{S}_{n}^{-1}$ in the second diagonal, this impedance matrix is antisymmetric (with a change in the orientation of the velocities at the extremity $n$, the impedance matrix would become symmetric). Notice that the matrix $\mathcal{Z}$ has the dimension of a specific impedance, while the matrix $\mathcal{G}$ has the dimension of the inverse of an area. Furthermore, for evanescent modes (real $\Gamma$ ) the ratios $\cosh n \Gamma / \sinh n \Gamma$ and $1 / \sinh n \Gamma$ do no diverge when $n$ tends to infinity, unlike the coefficients of the transfer matrix.

\subsection{Lattice of finite length inserted into an infinite waveguide}

We consider the geometry shown in Figure 4. A lattice of finite length, with $n$ cells, is inserted into an infinite

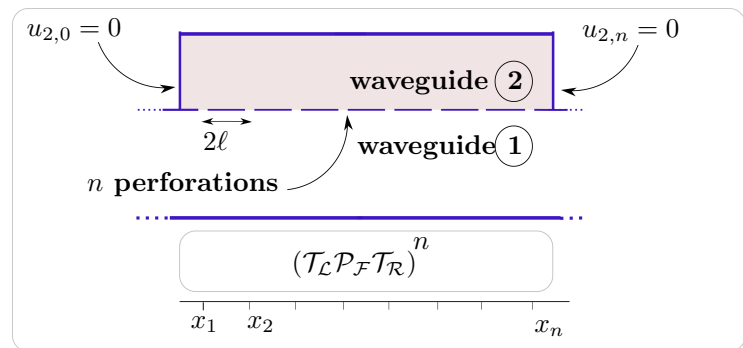

Figure 4: Lattice with $n$ cells, with the transfer ma$\operatorname{trix}\left(\mathcal{T}_{\mathcal{L}} \mathcal{P}_{\mathcal{F}} \mathcal{T}_{\mathcal{R}}\right)^{n}$ and boundary conditions for Guide 2 (see Equations (36 and 40)), inserted into an infinite waveguide.

waveguide so as to act as an acoustic wall treatment. By closing Guide 2 at each end of the lattice (Equation (36)) by an impedance condition (Figure 4), the 4thorder impedance matrix (Equation (35)) is reduced to a 2nd-order one, and the transmission coefficient of the finite lattice can be obtained.

Simple boundary conditions are chosen. Guide 2 is closed at each end by setting $u_{2,0}=u_{2, n}=0$ into Equation (35). The 2nd-order impedance matrix of the finite lattice can be derived:

$$
\begin{aligned}
\left(\begin{array}{c}
p_{1,0} \\
p_{1, n}
\end{array}\right) & =\left(\begin{array}{c}
Z_{A}-Z_{B} \\
Z_{B}-Z_{A}
\end{array}\right)\left(\begin{array}{c}
u_{1,0} \\
u_{1, n}
\end{array}\right), \text { with } \\
Z_{A} & =\left[\begin{array}{c}
{\left[\mathbf{Z C}_{n} \mathbf{S}_{n}^{-1} \mathbf{G}^{-1}\right]_{11}} \\
\end{array}=\left[\hat{Z} \operatorname{coth} n \Gamma+\hat{Z}^{\prime} \operatorname{coth} n \Gamma^{\prime}\right]\right. \\
Z_{B} & =\left[\hat{Z} \mathbf{S}_{n}^{-1} \mathbf{G}^{-1}\right]_{11} \\
& =\left[\hat{Z} / \sinh n \Gamma+\hat{Z}^{\prime} / \sinh n \Gamma^{\prime}\right],
\end{aligned}
$$

where the impedances $\hat{Z}$ and $\hat{Z}^{\prime}$ associated to each mode result from Equation (32) as follows:

$$
\hat{Z}=z_{1} g_{2}^{\prime} /(\operatorname{det} \mathbf{G}) \text { and } \quad \hat{Z}^{\prime}=z_{1}^{\prime} g_{2} /(\operatorname{det} \mathbf{G}) .
$$

Recall that these impedances are acoustic impedances (ratio pressure/flow rate). This particular case of lattice is built as the combination of two four-terminals with their extremities in series, each four-terminal corresponding to a propagation mode with constant $\Gamma$ and $\Gamma^{\prime}$. Expression (36) is then written in form of a transfer matrix:

$$
\begin{aligned}
\left(\begin{array}{l}
p_{1,0} \\
u_{1,0}
\end{array}\right) & =\left(\begin{array}{ll}
A_{s} & B_{s} \\
C_{s} & A_{s}
\end{array}\right)\left(\begin{array}{l}
p_{1, n} \\
u_{1, n}
\end{array}\right) \\
& =\frac{1}{Z_{B}}\left(\begin{array}{cc}
Z_{A} & \left(Z_{A}^{2}-Z_{B}^{2}\right) \\
1 & Z_{A}
\end{array}\right)\left(\begin{array}{l}
p_{1, n} \\
u_{1, n}
\end{array}\right) .
\end{aligned}
$$

Let us consider an infinite waveguide with characteristic impedance $\bar{z}_{c 1}=\rho_{1} c_{1} / S_{1}$. The outgoing and incoming plane wave have the amplitudes $p_{1}^{+}=$ 
$\left(p_{1}+\bar{z}_{c 1} u_{1}\right) / 2$ and $p_{1}^{-}=\left(p_{1}-\bar{z}_{c 1} u_{1}\right) / 2$, respectively.

Once the finite lattice of Fig.(4) is inserted, the trans- ${ }_{430}$ mission coefficient can be written as:

$$
\begin{aligned}
T & =\frac{p_{1, n}^{+}}{p_{1,0}^{+}}=\frac{p_{1,0}^{-}}{p_{1, n}^{-}}=\frac{2}{2 A_{s}+\left(B_{s} / \bar{z}_{c 1}+C_{s} \bar{z}_{c 1}\right)} \\
& =\frac{2 Z_{B} \bar{z}_{c 1}}{\left(Z_{A}+\bar{z}_{c 1}-Z_{B}\right)\left(Z_{A}+\bar{z}_{c 1}+Z_{B}\right)} .
\end{aligned}
$$

We notice from Expressions (42) that when $Z_{B}=0$ (Equation (38)), i.e., when:

$$
\hat{Z} / \sinh n \Gamma+\hat{Z}^{\prime} / \sinh n \Gamma^{\prime}=0,
$$

the transmission coefficient is zero.

\section{Analysis of the coupling ef- fect; local vs non-local reac- tion}

\subsection{Effect of the series impedance on the coupling}

${ }_{410}$ The respective roles of the series impedance $Z_{a}$ and the shunt admittance $Y_{s}$ can be discussed qualitatively at the zero-frequency limit. Exact values are known for the 2D, rectangular case. For the cylindrical case, we use approximate values for two guides with radii $a_{1}$ and $a_{2}$ and the same fluid density $\rho$, which exhibit the dependence on the parameters [28]:

$$
Y_{s}^{-1} \simeq j \omega \frac{\rho}{r_{p}} S_{1} ; Z_{a} \simeq-j \omega 0.57 \rho r_{p}^{2} a_{1} / S_{1},
$$

where $j=(-1)^{2}, \omega$ is the angular frequency. $r_{p}$ is the radius of the perforation, and $a_{2}$ is assumed to be larger than $a_{1}$. A first observation is that the product $Y_{s} Z_{a}$ is independent of the frequency and is very small, because it is proportional to $\left(r_{p} / a_{1}\right)^{3}$. As a consequence, at a first approximation, Equation (23) can 440 be simplified in:

$$
\begin{array}{r}
\left(\cosh \Gamma-A_{1}-\gamma_{2} E_{1}\right)\left(\cosh \Gamma-A_{2}-\gamma_{1} E_{2}\right) \\
=\gamma_{1} \gamma_{2} E_{1} E_{2} .
\end{array}
$$

The influence of the series impedance can be estimated by considering the expression of the quantities $E_{i}$. Considering the low frequency case, the guides are reduced to lumped elements, $B_{i}=2 j \omega \rho \ell$ is a mass and $C_{i}=2 j \omega \ell / \rho c^{2}$ is a compliance ( $c$ is the sound speed). It turns out that $Y_{s} B_{i}$ is a ratio of two masses, while $Z_{a} C_{i}$ is proportional to $\omega^{2}$ : therefore the effect of the series impedance $Z_{a}$ can be neglected 445 at low frequency. This justifies the following analysis of the coupling of the two guides with $Z_{a}=0$. This approximation will be done from here until to the end of the paper.

\subsection{Eigenvalues and eigenvectors for the simplified model}

What are the conditions for reducing the number of guided modes form 2 to 1 ?

If $Z_{a}=0$, the perforation matrix (Equation (4)) connects the two guides through one coupling quantity only, the shunt admittance $Y_{s}$. The dispersion Equation (16 or 23) reduces to Equation (45), with $E_{i}=Y_{s} B_{i}$. This equation is obtained for the choice of specific admittances and impedances, corresponding to the choice of acoustic pressure and velocity (of the planar mode) as basic quantities for the considered 4ports. This choice is convenient for the description of the perforation effects, but when the series impedance $Z_{a}$ is ignored, it is easier to use flow rates instead of velocities (therefore to use acoustic admittances and impedances). For this purpose the impedances and admittances need to be modified, and Equation (45) becomes:

$$
\begin{aligned}
\left(\cosh \Gamma-A_{1}-\frac{1}{2} \bar{Y}_{p} \bar{B}_{1}\right)(\cosh \Gamma & \left.-A_{2}-\frac{1}{2} \bar{Y}_{p} \bar{B}_{2}\right) \\
& =\frac{1}{4} \bar{Y}_{p}^{2} \bar{B}_{1} \bar{B}_{2},
\end{aligned}
$$

where $\bar{B}_{1}=B_{1} / S_{1} \quad \bar{B}_{2}=B_{2} / S_{2}$ and the acoustic admittance $\bar{Y}_{p}$ is given by:

$$
Y_{s}=\frac{1}{2}\left(\frac{1}{S_{1}}+\frac{1}{S_{2}}\right) \bar{Y}_{p}
$$

The bars above the symbols indicate acoustic impedances or admittances. When the radius of the perforation is very small, a simple formula can be chosen:

$$
\frac{1}{\bar{Y}_{p}}=\frac{j \omega\left(\rho_{1}+\rho_{2}\right)}{4 r_{p}}
$$

where $r_{p}$ is the radius of a circular perforation or the equivalent radius when the perforation is not circular. Another form of the dispersion equation is useful:

$$
\frac{2}{\bar{Y}_{p}}=\frac{\bar{B}_{1}}{\cosh \Gamma-A_{1}}+\frac{\bar{B}_{2}}{\cosh \Gamma-A_{2}} .
$$

For the calculation of the eigenvectors, we make the choice of an asymmetric cell, and use Equations (A17) and (A18). For the eigenvalue $\lambda=\exp (\Gamma)$, it is found:

$$
\mathbf{W}_{L 1}^{(i)}=\frac{v_{0}}{\gamma_{1}\left(\cosh \Gamma-A_{1}\right)}\left(\begin{array}{c}
\bar{B}_{1} S_{1} e^{-\Gamma} \\
1-A_{1} e^{-\Gamma}
\end{array}\right)
$$

and similarly for Guide 2 (with a change in sign). Using Equation (49), the pressure ratio is found to be:

$$
\frac{p_{1}}{p_{2}}=1+\frac{A_{2}-\cosh \Gamma}{\frac{1}{2} \bar{Y}_{p} \bar{B}_{2}} .
$$




\subsection{Definition of the coupling coeffi- cient}

The discriminant $\Delta$ of the quadratic equation in $\cosh \Gamma$ (Equation (46)) can be written by exhibiting a coupling coefficient $\mathcal{C}$, as follows:

$$
\begin{aligned}
& \Delta=\left(A_{1}-A_{2}\right)^{2}\left[1+2 \frac{\bar{B}_{1}-\bar{B}_{2}}{\bar{B}_{1}+\bar{B}_{2}} \mathcal{C}+\mathcal{C}^{2},\right] \\
& \text { where } \mathcal{C}=\frac{1}{2} \bar{Y}_{p} \frac{\bar{B}_{1}+\bar{B}_{2}}{A_{1}-A_{2}}
\end{aligned}
$$

The coupling coefficient $\mathcal{C}$ is proportional to the perforation admittance $\bar{Y}_{p}$, and inversely proportional to the difference between the coefficients $A_{1}$ and $A_{2}$ of ${ }_{480}$ the two guides, which are characteristic of the propagation into each guide separately. For identical guides, $\mathcal{C}$ is infinite; at low frequencies, the admittance is large, so is $\mathcal{C}$. Two extreme cases can therefore be distinguished.

\subsubsection{The weak coupling limit}

When the coupling coefficient $\mathcal{C}$ is small, the following solution is found:

$$
\cosh \Gamma=A_{1}+\frac{1}{2} \bar{Y}_{p} \bar{B}_{1}+\mathcal{C}^{2} \frac{\left(A_{1}-A_{2}\right) \bar{B}_{1} \bar{B}_{2}}{\left(\bar{B}_{1}+\bar{B}_{2}\right)^{2}}+O\left(\mathcal{C}^{3}\right)_{490}
$$

$$
\begin{aligned}
=A_{1} & +\frac{1}{2} \bar{Y}_{p} \overline{B_{1}}+\frac{1}{4} \frac{\bar{Y}_{p}^{2} \bar{B}_{1} \bar{B}_{2}}{A_{1}-A_{2}}+O\left(\mathcal{C}^{3}\right) . \\
\frac{p_{2}}{p_{1}} & =2 \mathcal{C}^{2} \frac{\left(A_{2}-A_{1}\right) B_{2}}{\left(\bar{B}_{1}+\bar{B}_{2}\right)^{2} \bar{Y}_{p}}+O\left(\mathcal{C}^{3}\right) \\
& =-\mathcal{C} \frac{\bar{B}_{2}}{\left(\bar{B}_{1}+\bar{B}_{2}\right)}+O\left(\mathcal{C}^{3}\right) .
\end{aligned}
$$

The mode $\Gamma^{\prime}$ is obtained by exchanging the subscripts can be interpreted, at the first order of $\mathcal{C}$, as follows: the medium 2 acts as an equivalent impedance $Z_{e q}=$ $\bar{Y}_{p}^{-1}$ on the medium 1 . The pressure becomes very small in the medium 2.

\subsubsection{The strong coupling limit; locally react- ing impedance}

Strong coupling occurs when the media are not very different or when the perforation effect is strong (large opening of the perforation and/or low frequencies). be written as a series expansion with respect to $\mathcal{C}^{-1}$ :

$$
\begin{aligned}
\cosh \Gamma= & \frac{A_{1} \bar{B}_{2}+A_{2} \bar{B}_{1}}{\bar{B}_{1}+\bar{B}_{2}} \\
- & \frac{1}{\mathcal{C}} \frac{\bar{B}_{1} \bar{B}_{2}}{\left(\bar{B}_{1}+\bar{B}_{2}\right)^{2}}\left(A_{1}-A_{2}\right)\left[1+O\left(\frac{1}{\mathcal{C}}\right)\right] \\
\cosh \Gamma^{\prime}= & \frac{1}{2} \bar{Y}_{p}\left(\bar{B}_{1}+\bar{B}_{2}\right)+\frac{A_{1} \bar{B}_{1}+A_{2} \bar{B}_{2}}{\bar{B}_{1}+\bar{B}_{2}} \\
& +\frac{1}{\mathcal{C}} \frac{\bar{B}_{1} \bar{B}_{2}}{\left(\bar{B}_{1}+\bar{B}_{2}\right)^{2}}\left(A_{1}-A_{2}\right)\left[1+O\left(\frac{1}{\mathcal{C}}\right)\right] .
\end{aligned}
$$

The mode $\Gamma$ is an average value of the two propagation constants given by $\cosh \Gamma=A_{1}$ and $\cosh \Gamma=A_{2}$. The mode $\Gamma^{\prime}$ is a generalization of the flute mode (see [28]): it is strongly evanescent at low frequencies and for large perforations (large $\bar{Y}_{p}$ ). This mode was also given by Pierce [22] for the continuous case of a perforated tube muffler. The pressure ratios corresponding to the two modes $\Gamma$ and $\Gamma^{\prime}$ are:

$$
\begin{aligned}
& {\left[\frac{p_{1}}{p_{2}}\right]=1+\frac{1}{\mathcal{C}}+\frac{1}{\mathcal{C}^{2}} \frac{\bar{B}_{1}}{\bar{B}_{1}+\bar{B}_{2}}\left[1+O\left(\frac{1}{\mathcal{C}}\right)\right]} \\
& {\left[\frac{p_{1}}{p_{2}}\right]^{\prime}=-\frac{\bar{B}_{1}+\bar{B}_{2}}{\bar{B}_{2}}+\frac{1}{\mathcal{C}} \frac{\bar{B}_{1}}{\bar{B}_{2}}-\frac{1}{\mathcal{C}^{2}} \frac{\bar{B}_{1}}{\bar{B}_{1}+\bar{B}_{2}}\left[1+O\left(\frac{1}{\mathcal{C}}\right)\right] .}
\end{aligned}
$$

The first solution corresponds to a modified planar mode while for the second, the two pressures are opposite in phase, at least at low frequencies. For the case where the mode $\Gamma^{\prime}$ is strongly evanescent, only the first mode can be taken into account, and the effect of the second medium on the first one (or viceversa) can be represented by an equivalent impedance $Z_{e q}$, the second one being equivalent to a locally reacting medium. This impedance can be found from the following dispersion equation:

$$
\cosh \Gamma=A_{1}+\frac{1}{2} \bar{Z}_{e q}^{-1} \bar{B}_{1},
$$

obtained by searching for the eigenvalue of the periodic medium built with a cell described by the following transfer matrix:

$$
\left(\begin{array}{ll}
A_{1} & \bar{B}_{1} \\
\bar{C}_{1} & A_{1}
\end{array}\right)\left(\begin{array}{cc}
1 & 0 \\
\bar{Z}_{e q}^{-1} & 1
\end{array}\right) .
$$

From Equations $(56,59)$, the following equivalent impedance $Z_{e q}$ is found to be:

$$
\bar{Z}_{e q}=\frac{\bar{B}_{2}}{\bar{Y}_{p}\left(\bar{B}_{1}+\bar{B}_{2}\right)}\left[1+O\left(\frac{1}{\mathcal{C}}\right)\right]+\frac{\bar{B}_{1}+\bar{B}_{2}}{2\left(A_{2}-A_{1}\right)} .
$$

Obviously this concept of equivalent impedance is especially interesting if it does not depend on the first medium: this situation occurs if $\bar{B}_{1}<<\bar{B}_{2}$, and $A_{1}<<A_{2}$. As expected, it can be checked that these conditions of local reaction are fulfilled in particular when the cells of the second medium are uncoupled, 


\begin{tabular}{|c|c|}
\hline Nb of cells $n$ & 5 \\
\hline Cell length $(2 \ell)(m)$ & $2.1710^{-1}$ \\
\hline Cross section $S_{1}\left(m^{2}\right)$ & $3.1410^{-2}$ \\
\hline Cross section $S_{2}\left(m^{2}\right)$ & $3.4610^{-2}$ \\
\hline Perf. radius $r_{p}(m)$ & $3.910^{-2}$ \\
\hline Perf. open area ratio $\sigma_{p}$ & $3.510^{-2}$ \\
\hline Diaph. radius $r_{d}(m)($ Fig.5) & 0.105 and 0.104 \\
\hline Diaph. open area ratio $\sigma_{d}$ (Fig.5) & 1 and 0.98 \\
\hline Diaph. radius $r_{d}(m)($ Fig.6) & 0.018 and 0 \\
\hline Diaph. open area ratio $\sigma_{d}$ (Fig.6) & 0.03 and 0 \\
\hline
\end{tabular}

Table 1: Geometrical parameters of the finite lattice

e.g. thanks to closed walls between them. If these ${ }_{550}$ conditions are satisfied the equivalent impedance is the sum of the perforation impedance and the half of the input impedance of a cell of the second medium, closed by a rigid wall:

$$
\bar{Z}_{e q}=\frac{1}{\bar{Y}_{p}}+\frac{1}{2} \frac{\bar{B}_{2}}{A_{2}-1}
$$

and corresponds to Helmholtz resonators branched on Guide 1.

\section{Analysis of lattices with finite ${ }_{560}$ length}

\subsection{Definition of the geometries con- sidered}

We consider the geometries of finite length lattices shown in Figure 3. In particular we focus on parameters (see Table 1) that correspond to the strong coupling case (see Section 5.3.2). Therefore Guide 2 is strongly coupled to Guide 1 and acts as an acoustic wall treatment on Guide 1.

If the series impedance of the perforation is ignored, the perforation matrix (Equation (4)) is completely defined by choosing the specific shunt admittance $Y_{s}$. A simple formula, sufficient for our purpose is chosen for the acoustic admittance $\bar{Y}_{p}$, according to Equation (48) as follows:

$$
\frac{1}{\bar{Y}_{p}}=R+j \omega \rho / 2 r_{p}
$$

where $R=2 \sqrt{2 \eta \rho \omega}$ is a small resistive term [42] describing viscous losses near the perforation and $\eta$ is the shear viscosity of the fluid. This allows limiting the resonance heights. Equation (62) is valid for small perforations, and also for a length between 570 perforations sufficiently large in comparison with the transverse dimensions of the guide. This issue, which is important for the understanding of the transition between discrete and continuous descriptions, is discussed in [28]. For the sake of simplicity we consider 575 here that an equivalent radius exists (see e.g. [43]).
We define the radius $r_{p}$ of the perforation thanks to its open area ratio in one cell of Guide $1 \sigma_{p}=\pi r_{p}^{2} /\left(2 \ell a_{1}\right)$. The shunt admittance $\bar{Y}_{p}$ vanishes when the perforation is closed.

In air, the transfer matrix for the planar mode along one uncoupled portion of length $\ell$ is:

$$
\mathbf{T}=\left(\begin{array}{cc}
\cos (k \ell) & j Z_{c} \sin (k \ell) \\
j \sin (k \ell) / Z_{c} & \cos (k \ell)
\end{array}\right) .
$$

If propagation losses are ignored, $Z_{c}=\rho c$ and $k=\omega / c$ are the characteristic impedance and wavenumber of the medium (air) filling the guides 1 and 2 . For Guide 1 , the transfer matrix along length $\ell$ is: $\mathbf{T}_{1 R}=\mathbf{T}_{1 L}=$ $\mathbf{T}$, and $\mathbf{T}_{1}=\mathbf{T}^{2}$ for the length $2 \ell$ (Equation (7)). Inside Guide 2, we write for the length $\ell$ on the left of a perforation $\mathbf{T}_{2 L}=\mathbf{D}_{\mathbf{d}} \mathbf{T}$, and $\mathbf{T}_{2 R}=\mathbf{T} \mathbf{D}_{\mathbf{d}}$ on the right. Therefore $\mathbf{T}_{2}=\mathbf{T D}_{\mathbf{d}}^{\mathbf{2}} \mathbf{T}$ for the length $2 \ell$. The matrix

$$
\mathbf{D}_{\mathbf{d}}=\left(\begin{array}{cc}
1 & \bar{Z}_{d} / 2 \\
0 & 1
\end{array}\right)
$$

corresponds to the presence of a diaphragm within Guide 2, and introduces the non-homogeneity between the two guides. For the sake of simplicity, the (acoustic) impedance is $\bar{Z}_{d}=j \omega \rho S_{2} / 2 r_{d}\left(1-\sqrt{\sigma_{d}}\right)$, where $r_{d}$ is the opening radius of a diaphragm without thickness, and $\sigma_{d}=\left(r_{d} / a_{2}\right)^{2}$ is its open area ratio. This is a crude simplification of Fock's formula [44]. In what follows, we first consider the transition between homogeneous lattices to slightly nonhomogeneous lattices, then the transition between ducts with branched resonators and strongly nonhomogeneous lattices.

\subsection{From homogeneous to slightly non-homogeneous lattices}

Consider the homogeneous lattice shown in Figure 3c, where the same medium fills the waveguides 1 and 2 . No diaphragm is placed in Waveguide 2. The lattice is said to be homogeneous. The wavenumber and characteristic impedance are identical for the two waveguides, and $A_{1}=A_{2}=A=\cos (2 k \ell)$. Equation (49) gives the planar mode with constant $\Gamma$ and the flute mode with constant $\Gamma^{\prime}$ :

$$
\begin{cases}\cosh \Gamma & =\cos (2 k \ell), \\ \cosh \Gamma^{\prime} & =\cos (2 k \ell)+j \bar{Y}_{p} Z_{c}\left[\frac{1}{S_{1}}+\frac{1}{S_{2}}\right] \sin (2 k \ell) .\end{cases}
$$

They correspond to the first term of Equations (56) and (57), respectively (the coupling coefficient $\mathcal{C}$ is infinite). Their variation with frequency is shown in Figure 5 (top and center). The planar mode, with $\Gamma=2 j k \ell$, is not dispersive and is unaffected by the perforation admittance $\bar{Y}_{p}$. The flute mode, with constant $\Gamma^{\prime}$, is evanescent at low frequencies, because $\bar{Y}_{p}$ is high, yielding $\cosh \Gamma^{\prime}>1($ or $\operatorname{Re}(\Gamma)>0$ and 

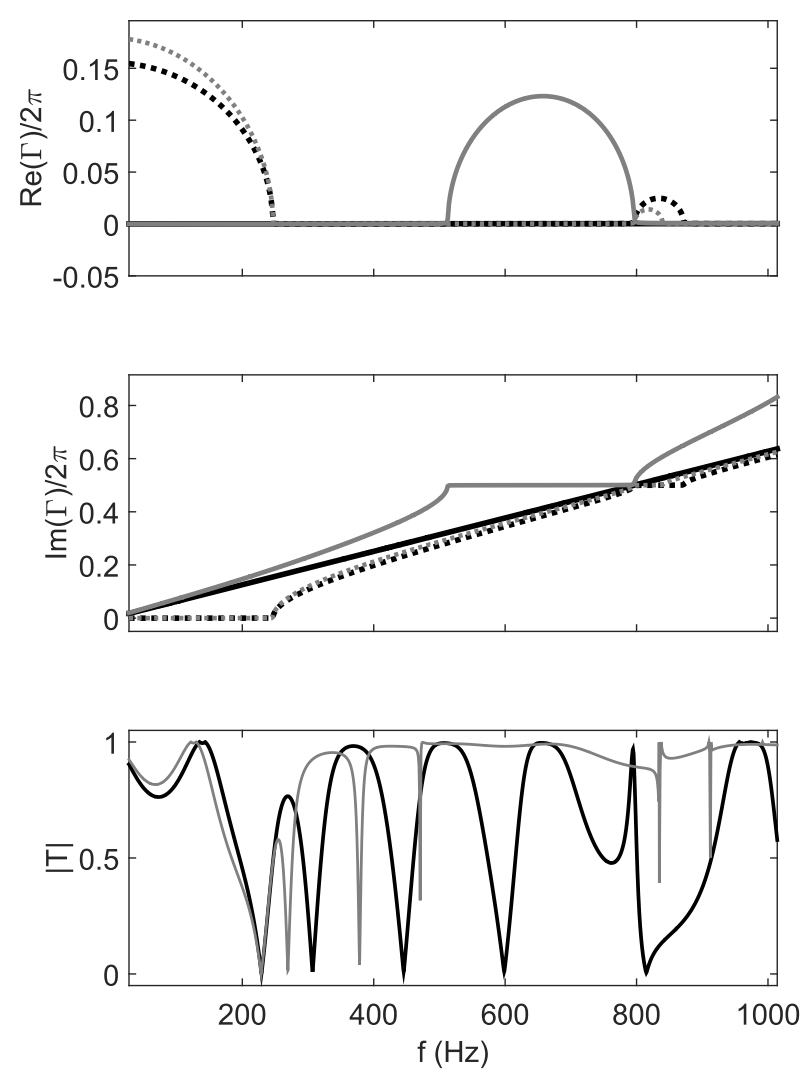

Figure 5: Dispersion curves $\Gamma(\omega)$ (top and center). Black lines: homogeneous lattice $\left(\sigma_{d}=1\right)$. Grey lines: slightly non-homogeneous lattice $\left(\sigma_{d}=0.98\right)$. Solid lines: mode with propagation constant $\Gamma$. Dotted lines: mode with propagation constant $\Gamma^{\prime}$ (notice that $\Gamma^{\prime}$ is mildly affected by the non-homogeneity). Transmission coefficient $|T(\omega)|$ (bottom) for 5 cells. Black line: homogeneous lattice $\left(\sigma_{d}=1\right)$ and Grey ${ }^{615}$ line: slightly non-homogeneous lattice $\left(\sigma_{d}=0.98\right)$.

$\operatorname{Im}(\Gamma)=0)$. The mode $\Gamma^{\prime}$ is cut on at $f=247 \mathrm{~Hz}$. Above this frequency the two modes propagate within ${ }_{620}$ the lattice.

Looking at frequencies below $797 \mathrm{~Hz}$ (this limit is explained later on), the transmission coefficient obtained for the homogeneous lattice (Figure 5 (bottom) is similar to the results presented in [1] (Insertion 625 Loss, Figure 12). The transmission coefficient curve exhibits a low frequency behaviour similar to that of an expansion chamber (driven by the expansion ratio $S_{1} / S_{2}$ ). At higher frequencies (between 247 and 797 $\mathrm{Hz}$ ), transmission coefficient minima are observed.

As a first result of the present analysis, this behaviour change in transmission coefficient at $247 \mathrm{~Hz}$ can be associated to the number of propagating modes (here 1 or 2) within the lattice. A second result is that the frequencies at which the transmission coefficient 635 is minimum are given by Equation (43). It can be

\begin{tabular}{|c|cc|}
\hline & \multicolumn{2}{|c|}{ Lattice mode } \\
\hline Freq.(Hz) & $\Gamma$ & $\Gamma^{\prime}$ \\
\hline $247^{*}$ & - & Eq.24 \\
\hline 509 & Eq.26 & - \\
\hline $797^{*}$ & Eq.27 & Eq.27 \\
\hline 839 & - & Eq.26 \\
\hline
\end{tabular}

Table 2: Cut-off frequencies of the slightly nonhomogeneous modes in Figure 5 (in grey). Symbol * denotes a cut-off frequency that does not depend on the diaphragm radius $r_{d}$.
595 shown that the minimum of the transmission coefficient is resonant (limited by losses) only if the two terms of Equation (43) have the same order of magnitude and if the lattice length is finite.

Above $797 \mathrm{~Hz}$, a stop band of Bragg type appears for the flute mode $\Gamma^{\prime}$, with a low transmission coefficient (Figure 5, bottom). In a Bragg stop band (or Bragg resonance due to spatial periodicity) $\operatorname{Im}(\Gamma)$ constant and equal to $n \pi$, where $n$ is an integer and $\operatorname{Re}(\Gamma)$ is positive but remains finite [40]. Unlike Fig${ }_{5}$ ure 5 , this behaviour is not reported in [1], presumably because the spatial periodicity of the lattice that [1] used makes the Bragg stop band out of the frequency band presented (unfortunately this periodicity is not mentioned in [1]).

Let us now consider a slightly non-homogeneous lattice. The diaphragms are now slightly closed $\left(\sigma_{d}=\right.$ $\left.\left(r_{d} / a_{2}\right)^{2} \simeq 0.98\right)$. The solution of the dispersion Equation (49) for the mode $\Gamma$ of the non-homogeneous lattice is:

$$
\cosh \Gamma=\frac{1}{2}\left(A_{1}+A_{2}+\frac{1}{2} \bar{Y}_{p}\left[\bar{B}_{1}+\bar{B}_{2}\right]-\sqrt{\Delta}\right),
$$

where $\Delta$ is given by Equation (52). The second solution for the mode $\Gamma^{\prime}$ is given by the same result, changing the sign before $\Delta$. The mode $\Gamma^{\prime}$ is not really affected by the added mass. In particular, its first cut-off frequency remains unchanged at $247 \mathrm{~Hz}$, like for the homogeneous lattice (the cut-off frequencies for the slightly non-homogeneous lattice are given in Table 2). This is explained by symmetry properties discussed in Section 3.2, which imply that the velocity within the diaphragms vanishes at that particular frequency.

However the mass added by the diaphragms strongly modifies the mode $\Gamma$ : the mode is now dispersive, with a phase velocity lower than that the planar mode of the homogeneous lattice (see Figure 5, center). This slowdown of the mode $\Gamma$ explains why the resonant minima of the transmission coefficient are shifted towards the low frequencies, compared to those of the homogeneous lattice. A Bragg stop band also appears for the mode $\Gamma$ between 509 and $797 \mathrm{~Hz}$ (see Figure 5, top): only the mode $\Gamma^{\prime}$ propagates and the transmission coefficient does not exhibit resonant 
peaks because the mode $\Gamma$ is evanescent. For the mode $\Gamma^{\prime}$, the width of the the Bragg stop band is reduced to the interval $[797,839] \mathrm{Hz}$, where the transmission coefficient is close to 1 . This issue could be further investigated.

In these simulations the number of cells of the lattice is limited to $n=5$ for the sake of readability of the transmission coefficent curves. Indeed, increasing $n$ increases the number of resonant peaks, according to Equation (43). But it can be checked that the computation does not encounter numerical difficulties even for high $n$ (and/or highly evanescent modes), thanks to the impedance matrix formalism.

Summarizing the effect of the non-homogeneity induced by the diaphragms, the width of frequency bands where two modes propagate is reduced, and therefore the possibility of resonant transmission coefficient minima as well.

\subsection{From ducts with branched Helmholtz resonators to strongly non-homogeneous lattices}

Let us now start from another classical muffler configuration: the branched Helmholtz resonators (Figure

$3 b)$, which are locally reacting. There is only one mode in the lattice. The model at its strong coupling limit (Equation (59)) tends to the following solution of the dispersion equation :

$$
\cosh \Gamma^{h}=\cos (2 k \ell)+j \frac{\rho c}{2 S_{1}} \bar{Z}_{e q}^{-1} \sin (2 k \ell)
$$

where, according to Equation (61), the input impedance of one resonator is $\bar{Z}_{e q}=1 / \bar{Y}_{p}-$ $j\left(\rho c / 2 S_{2}\right) \cot (k \ell) . \quad \Gamma^{h}$ is the classical Helmholtz mode. It is strongly dispersive (see Figure 6) and exhibits a stop band within the band $\left[f_{1}^{h}, f_{2}^{h}\right]=$ $[162,247] \mathrm{Hz}$, which can be called "Helmholtz stop band". This band is a resonance stop band (indeed, the Helmholtz resonance frequency $f_{r}$ is given by $\left.\bar{Z}_{e q}=0\right) \cdot \operatorname{Im}(\Gamma)=m \pi$ below $f_{r}$, and $\operatorname{Im}(\Gamma)=m^{\prime} \pi$ above $f_{r}, m$ being an even integer and $m^{\prime}$ an odd integer (or vice versa) [40]. Moreover, if there are no losses, $\operatorname{Re}(\Gamma)$ is infinite at $f_{r}$. The lower bound of the stop band $f_{1}^{h}$, given by $\cosh \Gamma^{h}=-1$ or

$$
\frac{2}{\bar{Y}_{p}}=j Z_{c}\left(-\frac{1}{S_{1}} \tan (k \ell)+\frac{1}{S_{2}} \cot (k \ell)\right) .
$$

The upper bound is $f_{2}^{h}$, given by $\cosh \Gamma^{h}=1$ or

$$
\frac{2}{\bar{Y}_{p}}=j Z_{c}\left(\frac{1}{S_{1}}+\frac{1}{S_{2}}\right) \tan (k \ell) .
$$

Notice that this cut-off frequency $f_{2}^{h}$ defined by 695 Equation (69) is the the first cut-off frequency of the
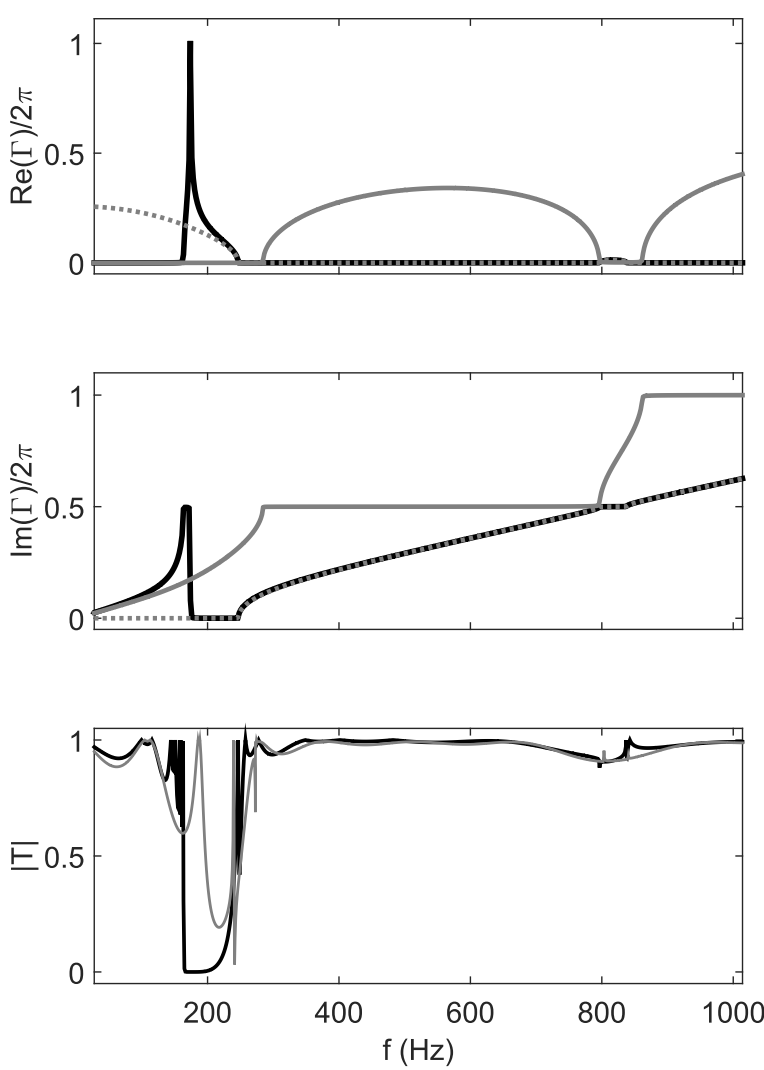

Figure 6: Dispersion curves $\Gamma(\omega)$ (top and center). Black line: branched Helmholtz resonators $\left(\sigma_{d}=0\right.$, notice that for non-coupled resonators, there is only one mode). Grey lines: strongly non-homogeneous lattice $\left(\sigma_{d}=0.03\right)$. Grey, solid line: mode with constant $\Gamma$. Grey, dotted line: mode with constant $\Gamma^{\prime}$. Transmission coefficient $|T(\omega)|$ (bottom) for 5 cells. Black line: branched Helmholtz resonators $\left(\sigma_{d}=0\right)$. Grey line: strongly non-homogeneous lattice $\left(\sigma_{d}=0.03\right)$.

remains unchanged when a diaphragm is open between resonators, because the velocity within the diaphragms vanishes at this particular frequency (Section 3.2).

Consider now Figure 6, which shows the effect of a strong inhomogeneity of the lattice. It appears that the mode $\Gamma^{\prime}\left(\sigma_{d}=\left(r_{d} / a_{2}\right)^{2} \approx 0.03\right)$, is cut off exactly at the upper bound of the Helmholtz stop band $f_{2}^{h}$. The cut-off frequencies for the strongly non-homogeneous lattice are given in Table 3 . The mode $\Gamma$ propagates at low frequencies and is evanescent for $f$ lying within $[284,797] \mathrm{Hz}$. This implies that even a small opening of the diaphragms between resonators entails that the stop band of the branched Helmholtz resonators disappears. In particular, the singularity of $\operatorname{Re}\left(\Gamma^{h}\right)$ at $f_{r}$, which is a characteristic of the Helmholtz stop band, is lost. 


\begin{tabular}{|c|cc|}
\hline & \multicolumn{2}{|c|}{ Lattice Mode } \\
\hline Freq.(Hz) & $\Gamma$ & $\Gamma^{\prime}$ \\
\hline $247^{*}$ & - & Eq.24 \\
\hline 284 & Eq.26 & - \\
\hline $797^{*}$ & Eq.27 & Eq.27 \\
\hline 839 & - & Eq.26 \\
\hline 861 & Eq.25 & - \\
\hline
\end{tabular}

Table 3: Cut-off frequencies of strongly nonhomogeneous modes in Figure 6 (in grey). Symbol

* denotes a cut-off frequency that does not depend on 750 the diaphragm radius $r_{d}$.

Summarizing the effect of the coupling of the branched resonators, the two modes have very different behaviours for all the frequencies considered. This tends to increase the transmission coefficient of the strongly non-homogeneous lattice compared to that of branched resonators.

\section{Conclusion} a wide variety of periodically coupled waveguides. For two classical examples of applications (homogeneous lattices and branched Helmholtz resonators), the model shows how the frequency behaviour of the transmission coefficient, can be attributed either to the properties of the medium (dispersion within the ${ }^{765}$ lattice), or to the boundary conditions and the finite length. Moreover, the introduction of a nonhomogeneity within the lattice, by means of an added properties (dispersion and transmission coefficient) of the two classical examples are modified, and how this can be interpreted.

A coupling coefficient is useful for the study of the transition between local and non-local reaction of one waveguide to the other. In practice, the model have shown that a very small coupling between (locally reacting) Helmholtz resonators is sufficient to obtain a lattice where the local reaction vanishes. A particular

Bragg and Helmholtz stop bands occurs. How this could be combined with finite length effects for sound attenuation purpose could be further investigated.

Other types of non-homogeneity, like the presence ${ }_{770}$ of dissipative media (porous materials described as equivalent fluids) or varying cross sections are in the scope of the method, provided that coupling of the evanescent modes created by two singularities does not occur, i.e., perforations are sufficiently spaced.

Arguments can be found for ignoring the series impedance of the perforation, but this restricts applications to cases where the frequency is low and the 775 perforation radius is small compared to the waveguides radii. The knowledge of appropriated expres- sions for series impedance and shunt admittance of the perforation would be required for practical application of the model to a particular geometry. An issue of interest could be the effects of the series impedance $Z_{a}$ on the properties of a finite lattice at higher frequencies. Precise values of the perforation admittance and impedance remain a topic of further investigation, in particular when the frequency increases. This can be done either with numerical methods or with measurements.

Application can be done to different kind of devices, such a silencers or sample of 1D metamaterials of finite length. To a certain extent, it could be possible to divide their design into two steps: first an optimization of the transmission coefficient with respect to given values of the perforation parameters, then a determination of the geometry corresponding to these parameters.

With the same model, further investigation could be done on dissipation effects, either in the perforations or in the waveguides. Mean flow or nonlinear effects would require different models.

\section{Appendix A : Derivation of the eigenvalues and eigenvectors of the transfer matrix}

\section{A1 Eigenvalues, dispersion equation}

For the sake of simplicity, the eigenvalues $\lambda$ and eigenvectors, denoted $\mathcal{W}_{L}$, are first sought for a generic, asymmetric cell. They are solutions of the 4th-order equation:

$$
\left(\mathcal{P}_{\mathcal{F}} \mathcal{T}-\lambda \mathcal{I}\right) \mathcal{W}_{L}=\mathcal{O}
$$

where $\mathcal{O}$ is the zero matrix of 4 th-order. Using Equations (2) and (7), and a calculation based upon submatrices, Equation (A1) can be rewritten as follows:

$$
\begin{aligned}
& \left\{\begin{array}{c}
\left.\left[\gamma_{1}+\gamma_{2} \mathbf{M}\right) \mathbf{T}_{1}-\lambda \mathbf{I}\right] \mathbf{W}_{L 1}+\gamma_{2}(\mathbf{I}-\mathbf{M}) \mathbf{T}_{2} \mathbf{W}_{L 2}=\mathbf{0} \\
\gamma_{1}(\mathbf{I}-\mathbf{M}) \mathbf{T}_{1} \mathbf{W}_{L 1}+\left[\left(\gamma_{2}+\gamma_{1} \mathbf{M}\right) \mathbf{T}_{2}-\lambda \mathbf{I}\right] \mathbf{W}_{L 2}=\mathbf{0}
\end{array}\right. \\
& \text { if } \quad \mathcal{W}_{L} \stackrel{\text { def }}{=} t\left(\mathbf{W}_{L 1} \mathbf{W}_{L 2}\right) .
\end{aligned}
$$

Subtracting the two Equations (A2) leads to a new equation:

$$
\left(\mathbf{M T}_{1}-\lambda \mathbf{I}\right) \mathbf{W}_{L 1}=\left(\mathbf{M} \mathbf{T}_{2}-\lambda \mathbf{I}\right) \mathbf{W}_{L 2} .
$$

Then, multiplying in System (A2) the first equation by $\gamma_{1}$ and the second equation by $\gamma_{2}$, and adding the resulting equations, the following equation is obtained:

$$
\gamma_{1}\left(\mathbf{T}_{1}-\lambda \mathbf{I}\right) \mathbf{W}_{L 1}=-\gamma_{2}\left(\mathbf{T}_{2}-\lambda \mathbf{I}\right) \mathbf{W}_{L 2} \stackrel{\text { def }}{=} \mathbf{W}_{0} .
$$


Then, writing for $i=1$ and 2 :

$$
\mathbf{M} \mathbf{T}_{i}-\lambda \mathbf{I}=\mathbf{M}\left(\mathbf{T}_{i}-\lambda \mathbf{I}\right)+\lambda(\mathbf{M}-\mathbf{I}),
$$

and substituting in Equation (A4) the values of $\mathbf{W}_{0}$ given by Equation (A5), Equation (A4) can be written as follows:

$$
\begin{aligned}
& (\mathbf{M}-\mathbf{I})+\mathbf{I}+ \\
& \lambda(\mathbf{M}-\mathbf{I})\left[\gamma_{2}\left(\mathbf{T}_{1}-\lambda \mathbf{I}\right)^{-1}+\gamma_{1}\left(\mathbf{T}_{2}-\lambda \mathbf{I}\right)^{-1}\right] \mathbf{W}_{0}=0 .
\end{aligned}
$$

Finally, multiplying Equation (A6) by the matrix $2(\mathbf{M}-\mathbf{I})^{-1}=\mathbf{K}-\mathbf{I}$, where $\mathbf{K}$ is given by Equation (4), it is found that the 4th-order Equation (A1) is equivalent to the following 2 nd-order equation:

$$
\begin{gathered}
\mathbf{T}_{0} \mathbf{W}_{0}=\mathbf{0}, \text { where } \\
\mathbf{T}_{\mathbf{0}}=\mathbf{I}+\mathbf{K}+2 \lambda\left[\gamma_{2}\left(\mathbf{T}_{1}-\lambda \mathbf{I}\right)^{-1}+\gamma_{1}\left(\mathbf{T}_{2}-\lambda \mathbf{I}\right)^{-1}\right] .
\end{gathered}
$$

Consequently, each eigenvalue $\lambda^{(i)}(i=1 . .4)$ is solution of the general dispersion equation:

$$
\operatorname{det} \mathbf{T}_{0}=A_{0} D_{0}-B_{0} C_{0}=0 .
$$

Here the coefficients of the matrix $\mathbf{T}_{0}$ are denoted $A_{0}$,

$B_{0}, C_{0}, D_{0}$. Equation (A8) gives their expression, which depends on the eigenvalue $\lambda$ :

$$
\begin{aligned}
& A_{0}=1+2 \lambda\left[\frac{\gamma_{2}\left(D_{1}-\lambda\right)}{\Delta_{1}}+\frac{\gamma_{1}\left(D_{2}-\lambda\right)}{\Delta_{2}}\right],( \\
& D_{0}=1+2 \lambda\left[\frac{\gamma_{2}\left(A_{1}-\lambda\right)}{\Delta_{1}}+\frac{\gamma_{1}\left(A_{2}-\lambda\right)}{\Delta_{2}}\right],( \\
& B_{0}=\frac{1}{Y_{s}}-2 \lambda\left[\gamma_{2} \frac{B_{1}}{\Delta_{1}}+\gamma_{1} \frac{B_{2}}{\Delta_{2}}\right] \\
& C_{0}=\frac{1}{Z_{a}}-2 \lambda\left[\gamma_{2} \frac{C_{1}}{\Delta_{1}}+\gamma_{1} \frac{C_{2}}{\Delta_{2}}\right]
\end{aligned}
$$

where the coefficients $A_{1,2}, B_{1,2}, C_{1,2}, D_{1,2}$ of matrices $\mathbf{T}_{1,2}$ correspond to the diagonal blocks of the 4 thorder transfer matrix $\mathcal{T}$ (see Equations (6) and (7), and where

$\Delta_{1,2}=\operatorname{det}\left(\mathbf{T}_{1,2}-\lambda \mathbf{I}\right)=\lambda^{2}-\lambda\left(A_{1,2}+D_{1,2}\right)+\operatorname{det} \mathbf{T}_{1,2}$.

Equation (A9) is a simplification of the dispersion equation (46) given in [28] (see Equation of this reference). In the same reference, the expression of the 4th-order equation for the unknown $\lambda$ is given (see

\section{A2 Eigenvectors for an asymmetric cell}

The eigenvectors of the matrix $\mathcal{P}_{\mathcal{F}} \mathcal{T}$ (see Equation (15)) can be obtained thanks to Equation (A5) by de-

a constant multiplicative value. The following general form is chosen:

$$
\mathbf{W}_{0}^{(i)}=v_{0}\left(\begin{array}{c}
w_{0}^{(i)} \\
-1
\end{array}\right),(i=1 . .4)
$$

where $v_{0}$ is an arbitrary constant having the dimension of a velocity, and $w_{0}^{(i)}$ are impedances associated to eigenvalues $\lambda^{(i)}$. By construction, Expression (A15) fulfils Equation (A7), which means that for each eigenvalues $\lambda^{(i)}$ we have:

$$
\left.w_{0}^{(i)}=\frac{B_{0}^{(i)}}{A_{0}^{(i)}}=\frac{D_{0}^{(i)}}{C_{0}^{(i)}}(i=1 . .4)\right) .
$$

Introducing the general form of $\mathbf{W}_{\mathbf{0}}$ (Equation (A15)) into Expression (A5) gives the eigenvectors of an asymmetric cell of the lattice. For the upper and lower rows, each column $i(i=1 . .4)$ of the matrix $\mathcal{E}_{\mathcal{L}}$ (Equation (15)) can be written as:

$$
\begin{aligned}
& \mathbf{W}_{L 1}^{(i)}=\frac{v_{0}}{\gamma_{1} \Delta_{1}}\left(\begin{array}{c}
w_{0}^{(i)}\left[D_{1}-\lambda^{(i)}\right]+B_{1} \\
-w_{0}^{(i)} C_{1}-\left[A_{1}-\lambda^{(i)}\right]
\end{array}\right),( \\
& \mathbf{W}_{L 2}^{(i)}=-\frac{v_{0}}{\gamma_{2} \Delta_{2}}\left(\begin{array}{c}
w_{0}^{(i)}\left[D_{2}-\lambda^{(i)}\right]+B_{2} \\
-w_{0}^{(i)} C_{2}-\left[A_{2}-\lambda^{(i)}\right]
\end{array}\right)(
\end{aligned}
$$

Finally each column $i(i=1 . .4)$ of the matrix $\mathcal{E}_{\mathcal{L}}$ is obtained by assembling the 2nd-order vectors $\mathbf{W}_{L 1}^{(i)}$ and $\mathbf{W}_{L 2}^{(i)}$ by Equation (A3).

\section{A3 Eigenvectors for a symmetric cell}

The dispersion equation (A9) and the expression (A17,A18)) of the eigenvectors are general. A more useful expression can be obtained if the propagation matrix $\mathcal{T}$ is splitted into two matrices in order to get a symmetric cell (see Section 2.4 and Figure 2). Consequently $A_{0}=D_{0}$.

The transfer matrix of a symmetric cell (Equation (9)) is written in the diagonal form (Equation (13)):comparing this equation and Equation (13), the columns of the eigenvector matrix $\mathcal{E}$ (Equation (15)) can be obtained from the eigenvectors of the antisymmetric cell $\mathcal{W}_{L}^{(i)}$ (Equations $\left.(\mathrm{A} 17, \mathrm{~A} 18)\right)$ by:

$$
\mathcal{W}^{(i)}=\left(\begin{array}{l}
\mathbf{W}_{1}^{(i)} \\
\mathbf{W}_{2}^{(i)}
\end{array}\right)=\mathcal{T}_{L} \mathcal{W}_{L}^{(i)}
$$

Therefore, using Equation (A5), $\mathbf{W}_{1}^{(i)}$ is given by:

$\mathbf{W}_{1}^{(i)}=\frac{1}{\gamma_{1}}\left(\mathbf{T}_{R 1}-\lambda \mathbf{T}_{L 1}^{-1}\right)^{-1} \mathbf{W}_{0}^{(i)}$ or

$\mathbf{W}_{1}^{(i)}=\frac{v_{0}}{\Delta_{1} \gamma_{1}}\left(\begin{array}{c}w_{0}^{(i)} D_{R 1}\left(\delta_{1}-\lambda^{(i)}\right)+B_{R 1}\left(\delta_{1}+\lambda^{(i)}\right) \\ -w_{0}^{(i)} C_{R 1}\left(\delta_{1}+\lambda^{(i)}\right)-A_{R 1}\left(\delta_{1}-\lambda^{(i)}\right)\end{array}\right)$

with $\delta_{1}=\operatorname{det}\left(\mathbf{T}_{L 1}\right)=\operatorname{det}\left(\mathbf{T}_{R 1}\right)$. A similar expression holds for the guide 2 , with a sign - before $v_{0}$. When the determinants are unity, the eigenvectors are given by Equation (18). 


\section{Appendix B: Transfer matrix of a lattice of $n$ cells}

\section{B1 First form of the transfer matrix}

In order to simplify the calculation of the invert matrix of $\mathcal{E}$ (Equation (18)), it is convenient to write its

first up-left quarter in the form of a matrix product:

$$
\left(\mathbf{W}_{1}^{(1)} \mathbf{W}_{1}^{(2)}\right)=v_{0} \mathbf{H}_{1} \mathbf{F D}^{-1 / 2}
$$

where $\mathbf{H}_{1}=\left(\begin{array}{cc}z_{1} & 0 \\ 0 & h_{1}\end{array}\right), \mathbf{F}=\left(\begin{array}{cc}1 & 1 \\ 1 & -1\end{array}\right)$ and

$\mathbf{D}=\left(\begin{array}{cc}e^{\Gamma} & 0 \\ 0 & e^{-\Gamma}\end{array}\right)$. Using similar notations, the three other quarters of the matrix $\mathcal{E}$ are:

$$
\begin{aligned}
\left(\mathbf{W}_{1}^{(3)} \mathbf{W}_{1}^{(4)}\right) & =v_{0} \mathbf{H}_{1}^{\prime} \mathbf{F D}^{\prime-1 / 2}, \\
\left(\mathbf{W}_{2}^{(1)} \mathbf{W}_{2}^{(2)}\right) & =-v_{0} \mathbf{H}_{2} \mathbf{F D}^{-1 / 2}, \\
\left(\mathbf{W}_{2}^{(3)} \mathbf{W}_{2}^{(4)}\right) & =-v_{0} \mathbf{H}_{2}^{\prime} \mathbf{F D}^{\prime-1 / 2} .
\end{aligned}
$$

By assembling expressions (B1-B4), the eigenvector matrix $\mathcal{E}=\left(\mathcal{W}^{(1)} \mathcal{W}^{(2)} \mathcal{W}^{(3)} \mathcal{W}^{(4)}\right)$ is written as:

$$
\mathcal{E}=v_{0}\left(\begin{array}{cc}
\tilde{\mathbf{H}}_{1} & \tilde{\mathbf{H}}_{1}^{\prime} \\
-\tilde{\mathbf{H}}_{2} & -\tilde{\mathbf{H}}_{2}^{\prime}
\end{array}\right)\left(\begin{array}{cc}
\mathbf{F} & \mathbf{0} \\
\mathbf{0} & \mathbf{F}
\end{array}\right)\left(\begin{array}{cc}
\mathbf{D} & \mathbf{0} \\
\mathbf{0} & \mathbf{D}^{\prime}
\end{array}\right)^{-1 / 2} .
$$

Thanks to this particular form for $\mathcal{E}$, that results from 855 reciprocity, the 4 th-order transfer matrix for a lattice of $n$ symmetric cells $\left(\mathcal{T}_{L} \mathcal{P}_{F} \mathcal{T}_{R}\right)^{n}=\left(\mathcal{E} \mathcal{D}^{n} \mathcal{E}^{-1}\right)$ is obtained as:

$$
\begin{aligned}
\left(\mathcal{T}_{L} \mathcal{P}_{F} \mathcal{T}_{R}\right)^{n} & =\mathcal{E} \mathcal{D}^{n} \mathcal{E}^{-1}=\left(\begin{array}{cc}
\mathbf{H}_{1} & \mathbf{H}_{1}^{\prime} \\
-\mathbf{H}_{2} & -\mathbf{H}_{2}^{\prime}
\end{array}\right)\left(\begin{array}{cc}
\mathbf{F} & \mathbf{0} \\
\mathbf{0} & \mathbf{F}
\end{array}\right){ }_{860} \\
& \left.\times\left(\begin{array}{cc}
\mathbf{D} & \mathbf{0} \\
\mathbf{0} & \mathbf{D}^{\prime}
\end{array}\right)^{n}\left(\begin{array}{cc}
\mathbf{F} & \mathbf{0} \\
\mathbf{0} & \mathbf{F}
\end{array}\right)^{-1}\left(\begin{array}{cc}
\mathbf{H}_{1} & \mathbf{H}_{1}^{\prime} \\
-\mathbf{H}_{2} & -\mathbf{H}_{2}^{\prime}
\end{array}\right){ }^{-1} \mathrm{~B} 6\right)
\end{aligned}
$$

\section{B2 Second form of the transfer matrix}

In order to derive the impedance matrix, a second ${ }^{865}$ form of the transfer matrix is useful. The vector $\mathcal{V}$ (Equation (1)) is replaced by a vector $\widetilde{\mathcal{V}}$ defined as follows:

$$
\widetilde{\mathcal{V}}=\left(\begin{array}{c}
\mathbf{P} \\
\mathbf{U}
\end{array}\right) \quad \text { where } \mathbf{P}=\left(\begin{array}{l}
p_{1} \\
p_{2}
\end{array}\right) \text { and } \mathbf{U}=\left(\begin{array}{l}
u_{1} \\
u_{2}
\end{array}\right),
$$

where $u_{i}=S_{i} v_{i}(i=1,2)$ are the flow rates. Considering the eigenvector matrix $\mathcal{E}$ (Equation (18)), a permutation of the second and third rows and columns is required, as well as a permutation of the second and third eigenvalues (see e.g. [45]). The following result is obtained:

$$
\begin{gathered}
\widetilde{\mathcal{E}}=v_{0}\left(\begin{array}{cccc}
z_{1} & z_{1}^{\prime} & z_{1} & z_{1}^{\prime} \\
-z_{2} & -z_{2}^{\prime} & -z_{2} & -z_{2}^{\prime} \\
g_{1} & g_{1}^{\prime} & -g_{1} & -g_{1}^{\prime} \\
-g_{2} & -g_{2}^{\prime} & g_{2} & g_{2}^{\prime}
\end{array}\right) \widetilde{\mathcal{D}}^{-1 / 2} \\
\widetilde{\mathcal{D}}=\left(\begin{array}{cccc}
e^{\Gamma} & 0 & 0 & 0 \\
0 & e^{\Gamma^{\prime}} & 0 & 0 \\
0 & 0 & e^{-\Gamma} & 0 \\
0 & 0 & 0 & e^{-\Gamma^{\prime}}
\end{array}\right)
\end{gathered}
$$

if $g_{i}=S_{i} h_{i}$, or equivalently:

$$
\begin{aligned}
& \widetilde{\mathcal{E}}=v_{0}\left(\begin{array}{cc}
\mathbf{Z} & \mathbf{0} \\
\mathbf{0} & \mathbf{G}
\end{array}\right)\left(\begin{array}{cc}
\mathbf{I} & \mathbf{I} \\
\mathbf{I} & -\mathbf{I}
\end{array}\right) \text { with } \\
& \mathbf{Z}=\left(\begin{array}{cc}
z_{1} & z_{1}^{\prime} \\
-z_{2} & -z_{2}^{\prime}
\end{array}\right), \mathbf{G}=\left(\begin{array}{cc}
g_{1} & g_{1}^{\prime} \\
-g_{2} & -g_{2}^{\prime}
\end{array}\right) .
\end{aligned}
$$

Finally the second from of the transfer matrix is:

$$
\widetilde{\mathcal{V}}_{0}=\left(\begin{array}{ll}
\mathbf{Z} & \mathbf{0} \\
\mathbf{0} & \mathbf{G}
\end{array}\right)\left(\begin{array}{ll}
\mathbf{C}_{\mathbf{n}} & \mathbf{S}_{\mathbf{n}} \\
\mathbf{S}_{\mathbf{n}} & \mathbf{C}_{\mathbf{n}}
\end{array}\right)\left(\begin{array}{ll}
\mathbf{Z} & \mathbf{0} \\
\mathbf{0} & \mathbf{G}
\end{array}\right)^{-1} \widetilde{\mathcal{V}}_{n}
$$

with $\mathbf{C}_{n}=\left(\begin{array}{cc}\cosh n \Gamma & 0 \\ 0 & \cosh n \Gamma^{\prime}\end{array}\right)$, and $\mathbf{S}_{n}=$ $\left(\begin{array}{cc}\sinh n \Gamma & 0 \\ 0 & \sinh n \Gamma^{\prime}\end{array}\right)$.

\section{Appendix C: Reciprocity rela- tionships}

Reciprocity is related to the choice of matrices $\mathbf{T}_{1}, \mathbf{T}_{2}$ and $\mathbf{M}$, with a determinant equal to unity. In order to find the consequences on the eigenvectors of a cell, we start from the classical reciprocity equation valid for guides without flow. We write it on the surface $\Sigma$ of a cell (e.g., a symmetric cell):

$$
\iint_{\Sigma}\left(p^{(i)} \overrightarrow{v^{(j)}}-p^{(j)} \overrightarrow{v^{(i)}}\right) d \vec{\Sigma}=0 .
$$

The superscripts $i$ and $j$ correspond to two different situations. For instance two situations where only one eigenmode exists can be chosen. The integral vanishes on all rigid walls, therefore it is limited to the input and output of a cell. The term in parenthesis in Equation (C1) is the same for the output surface and the input surface, apart from the factor $-\exp \left(\Gamma^{(i)}\right) \exp \left(\Gamma^{(j)}\right)$. Therefore it is possible to factorize the term $\left[1-\exp \left(\Gamma^{(i)}\right) \exp \left(\Gamma^{(j)}\right)\right]$, and for the eigenmodes corresponding to $\Gamma$ and $-\Gamma$, Equation (C1) is trivial because this term vanishes. It remains to solve the following equation:

$$
\iint_{S_{1}+S_{2}}\left(p \overrightarrow{v^{\prime}}-p^{\prime} \vec{v}\right) d \vec{\Sigma}=0
$$


for the modes corresponding to $\Gamma$ and $\Gamma^{\prime}$, and to $\Gamma$ and $-\Gamma^{\prime}$. Using the expressions (18) of the eigenvectors, 920 the following equations are obtained:

$$
\gamma_{1} z_{1} h_{1}^{\prime}=-\gamma_{2} z_{2} h_{2}^{\prime} ; \gamma_{1} z_{1}^{\prime} h_{1}=-\gamma_{2} z_{2}^{\prime} h_{2} \text {. }
$$

A direct checking of these equations is heavy. Reciprocity implies also the symmetry of the impedance 925 matrix, as shown in Section 4.1.

\section{Acknowledgments}

The authors thank Yves Aurégan for fruitful discussions during the preparation of the manuscript.

\section{References}

[1] J. W. Sullivan and M. J. Crocker. Analysis of concentrical tube resonators having un- ${ }^{935}$ partitioned cavities. J. Acoust. Soc. Am., 64 (1978)207-215.

[2] J. W. Sullivan. A method for modeling perforated tube muffler components. I. Theory. J. Acoust. Soc. Am., 66 (1979)772-778.

[3] A. Hasegawa and H. Hataoka. Acoustic TwoHole and Multi-Hole Directional Couplers. Acta Acust united Ac, 48 (1981) 158-167.

[4] K. Jayaraman and K. Yam. Decoupling approach 945 to modeling perforated tube muffler components. J. Acoust. Soc. Am., 69 (1981)390-396.

[5] Y. Aurégan and M. Leroux. Failures in the discrete models for flow duct with perforations: an experimental investigation. J. Sound Vib., 265950 (2003) 109-121.

[6] Xiang Yu, Li Cheng, and Xiangyu You. Hybrid silencers with micro-perforated panels and internal partitions. J. Acoust. Soc. Am., 137 (2015) 951-962.

\section{5}

[7] R. Kirby. A comparison between analytic and numerical methods for modelling automotive dissipative silencers with mean flow. J. Sound Vib., 325 (2009) 565-582.

[8] Z.L. Ji. Boundary element acoustic analysis of hybrid expansion chamber silencers with perforated facing. Eng. Anal. Boundary Elem., 34 (2010) 690-696.

[9] C. Jiang, T.W. Wu, and C.Y.R. Cheng. A single- ${ }_{965}$ domain boundary element method for packed silencers with multiple bulk-reacting sound absorbing materials. Eng. Anal. Boundary Elem., 34 (2010) 971-976.
[10] N. Fang, D. Xi, J. Xu, M. Ambati, W. Srituravanich, C. Sun, and X. Zhang. Ultrasonic metamaterials with negative modulus. Nat. Mater., 5 (2006) 452-456.

[11] Yan-Feng Wang, Vincent Laude, and Yue-Sheng Wang. Coupling of evanescent and propagating guided modes in locally resonant phononic crystals. J. Phys. D: Appl. Phys., 47(2014) 475502.

[12] S. Griffin, S. A. Lane, and S. Huybrechts. Coupled Helmholtz Resonators for Acoustic Attenuation. J. Vib. Acoust., 123 (2000) 11-17.

[13] R. Tagg and L. Faulkner. Multiple frequency optimization of coupled Helmholtz resonators for improved acoustic nacelle liners for turbofan engines. In 7th Aeroacoustics Conference. American Institute of Aeronautics and Astronautics, 1981.

[14] M. Jones, M. Tracy, W. Watson, and T. Parrott. Effects of Liner Geometry on Acoustic Impedance. In 8th $A I A A / C E A S$ Aeroacoustics Conference $\mathscr{G}$ Exhibit. American Institute of Aeronautics and Astronautics, 2002.

[15] S. H. Ko. Theoretical analyses of sound attenuation in acoustically lined flow ducts separated by porous splitters (rectangular, annular and circular ducts). J. Sound Vib., 39 (1975) 471-487.

[16] Y. Aurégan, A. Debray, and R. Starobinski. Low frequency sound propagation in a coaxial cylindrical duct: application to sudden area expansions and to dissipative silencers. J. Sound Vib., 243 (2001) 461-473.

[17] Hoi-Jeon Kim and Jeong-Guon Ih. Rayleigh Ritz approach for predicting the acoustic performance of lined rectangular plenum chambers. J. Acoust. Soc. Am., 120 (2006) 1859-1870.

[18] R. Kirby. The influence of baffle fairings on the acoustic performance of rectangular splitter silencers. J. Acoust. Soc. Am., 118 (2005) 23022312 .

[19] R. Kirby, P. T. Williams, and J. Hill. A three dimensional investigation into the acoustic performance of dissipative splitter silencers. J. Acoust. Soc. Am., 135 (2014) 2727-2737.

[20] R. Binois, E. Perrey-Debain, N. Dauchez, B. Nennig, J.M. Ville, and G. Beillard. On the Efficiency of Parallel Baffle Type Silencers in Rectangular Ducts: Prediction and Measurement. Acta Acust united Ac, 101 (2015) 520-530.

[21] K. S. Peat. A numerical decoupling analysis of perforated pipe silencer elements. J. Sound Vib., 123 (1988) 199-212. 
[22] A. D. Pierce. Acoustics: an introduction to its physical principles and applications. Acoustical Society of America, New-York, mcgraw-hill book1020 company, inc. edition, 1991.

[23] Dah-You Maa. Potential of microperforated panel absorber. J. Acoust. Soc. Am., 104 (1998) $2861-2866$.

[24] N. S. Dickey, A. Selamet, and M. S. Ciray. An ex- ${ }^{1025}$ perimental study of the impedance of perforated plates with grazing flow. J. Acoust. Soc. Am., 110 (2001) 2360-2370.

[25] K.S. Peat, R. Sugimoto, and J.L. Horner. The effects of thickness on the impedance of a rectan- ${ }^{10}$ gular aperture in the presence of a grazing flow. J. Sound Vib., 292 (2006) $610-625$.

[26] F. D. Denia, A. Selamet, F. J. Fuenmayor, and R. Kirby. Acoustic attenuation performance of ${ }_{1035}$ perforated dissipative mufflers with empty inlet/outlet extensions. J. Sound Vib., 302 (2007) 1000-1017.

[27] T. Bravo, C. Maury, and C. Pinhède. Optimisation of micro-perforated cylindrical silencers in $_{1040}$ linear and nonlinear regimes. J. Sound Vib., 363 (2016) :359-379.

[28] J. Kergomard, A. Khettabi, and X. Mouton. Propagation of acoustics waves in two waveguides coupled by perforations. I. Theory. Acta Acustica, https://hal.archives- ${ }^{1045}$ ouvertes.fr/hal-01260388, 2 (1994) 1-16.

[29] V. Dubos, J Kergomard, A. Khettabi, J.-P. Dalmont, D.H. Keefe, and C.J. Nederveen. Theory of sound propagation in a duct with a branched tube using modal decomposition. Acta Acustio50 united Ac, 85(1999) 153-169.

[30] C. J. Nederveen. Acoustical aspects of woodwind instruments. Northern Illinois University Press, 1998.

[31] V. Debut, J. Kergomard, and F. Laloë. Analysis and optimisation of the tuning of the twelfths for a clarinet resonator. Applied acoustics, 66 (2005) 365-409.

[32] Zhengyou Liu, Xixiang Zhang, Yiwei Mao, Y. Y. Zhu, Zhiyu Yang, C. T. Chan, and Ping Sheng. Locally Resonant Sonic Materials. Science, 2895485 (2000) 1734-1736.

[33] T. W. Wu, C. Y. R. Cheng, and P. Zhang. A direct mixed-body boundary element method for packed silencers. J. Acoust. Soc. Am., 111 (2002) 2566-2572.
[34] T. Kar and M. L. Munjal. Generalized analysis of a muffler with any number of interacting ducts. J. Sound Vib., 285 (2005) 585-596.

[35] Chien-Ho Wu and Chao-Nan Wang. Numerical Analysis of Parallel Perforated Single-Inlet and Double-Outlet Mufflers Using the Transfer Function. Acta Acust united Ac, 96(2010) 445-451.

[36] P. Y. Chen, R. C. McPhedran, C. M. de Sterke, C. G. Poulton, A. A. Asatryan, L. C. Botten, and M. J. Steel. Group velocity in lossy periodic structured media. Phys. Rev. A, 82(2010) 053825 .

[37] D. Lafarge and N. Nemati. Nonlocal maxwellian theory of sound propagation in fluid-saturated rigid-framed porous media. Wave Motion, 50 (2013) 1016-1035.

[38] J-P Groby, W Huang, A Lardeau, and Y Aurégan. The use of slow waves to design simple sound absorbing materials. J. Appl. Phys., 117(2015):124903.

[39] L. Brillouin. Wave Propagation in Periodic Structures. Dover Publications, Inc., New York, 2nd edition, 1953. p205.

[40] C. Elachi. Waves in active and passive periodic structures: A review. Proc. IEEE, 64 (1976) 1666-1698.

[41] J. S. Schwinger and D. S. Saxon. Discontinuities in Waveguides: Notes on Lectures by Julian Schwinger. CRC Press, January 1968.

[42] Uno Ingard. On the theory and design of acoustic resonators. J. Acoust. Soc. Am., 25 (1953) $1037-$ 1061.

[43] S. N. Rschevkin. The theory of sound. Pergamon press, 1963.

[44] W.A. Fock. A theoretical investigation of the acoustical conductivity of a circular aperture in a wall put across a tube. Comptes rendus de l'Académie des Sciences de I'U.R.S.S. (Doklady Akad. Nauk. SSSR), 31 (1941)875-878.

[45] G. Strang. Linear Algebra and Its Applications. Academic Press, May 2014. 